\title{
Recognition of DHN-melanin by a C-type lectin receptor is required for immunity to Aspergillus
}

\author{
Mark H. T. Stappers ${ }^{1 *}$, Alexandra E. Clark ${ }^{1 *}$, Vishukumar Aimanianda ${ }^{2}$, Stefan Bidula ${ }^{1}$, Delyth M. Reid ${ }^{1}$, \\ Patawee Asamaphan ${ }^{1}$, Sarah E. Hardison ${ }^{1}$, Ivy M. Dambuza ${ }^{1}$, Isabel Valsecchi ${ }^{2}$, Bernhard Kerscher ${ }^{1}$, Anthony Plato ${ }^{1}$, \\ Carol A. Wallace ${ }^{1}$, Raif Yuecel ${ }^{3}$, Betty Hebecker ${ }^{1}$, Maria da Glória Teixeira Sousa ${ }^{1}$, Cristina Cunha ${ }^{4,5}$, Yan Liu ${ }^{6}$, Ten Feizi ${ }^{6}$, \\ Axel A. Brakhage ${ }^{7}$, Kyung J. Kwon-Chung ${ }^{8}$, Neil A. R. Gow ${ }^{1}$, Matteo Zanda ${ }^{9}$, Monica Piras ${ }^{9}$, Chiara Zanato ${ }^{9}$, Martin Jaeger ${ }^{10}$, \\ Mihai G. Netea ${ }^{10}$, Frank L. van de Veerdonk ${ }^{10}$, João F. Lacerda ${ }^{11,12}$, António Campos Jr ${ }^{13}$, Agostinho Carvalho ${ }^{4,5}$, \\ Janet A. Willment ${ }^{1}$, Jean-Paul Latgé ${ }^{+} \uparrow$ \& Gordon D. Brown ${ }^{1}$
}

\begin{abstract}
Resistance to infection is critically dependent on the ability of pattern recognition receptors to recognize microbial invasion and induce protective immune responses. One such family of receptors are the $\mathrm{C}$-type lectins, which are central to antifungal immunity ${ }^{1}$. These receptors activate key effector mechanisms upon recognition of conserved fungal cell-wall carbohydrates. However, several other immunologically active fungal ligands have been described; these include melanin ${ }^{2,3}$, for which the mechanism of recognition is hitherto undefined. Here we identify a C-type lectin receptor, melanin-sensing C-type lectin receptor (MelLec), that has an essential role in antifungal immunity through recognition of the naphthalene-diol unit of 1,8-dihydroxynaphthalene (DHN)-melanin. MelLec recognizes melanin in conidial spores of Aspergillus fumigatus as well as in other DHN-melanized fungi. MelLec is ubiquitously expressed by $\mathrm{CD} 1^{+}$endothelial cells in mice, and is also expressed by a sub-population of these cells that co-express epithelial cell adhesion molecule and are detected only in the lung and the liver. In mouse models, MelLec was required for protection against disseminated infection with $A$. fumigatus. In humans, MelLec is also expressed by myeloid cells, and we identified a single nucleotide polymorphism of this receptor that negatively affected myeloid inflammatory responses and significantly increased the susceptibility of stem-cell transplant recipients to disseminated Aspergillus infections. MelLec therefore recognizes an immunologically active component commonly found on fungi and has an essential role in protective antifungal immunity in both mice and humans.
\end{abstract}

C-type lectin receptors (CLRs) involved in antifungal immunity belong primarily to the Dectin-1 (also known as CLEC7A) and Dectin-2 (also known as CLEC4N) clusters of receptors located near the natural killer gene complex ${ }^{1}$. To identify new CLRs within these clusters that recognize fungi, we generated soluble protein chimaeras consisting of the C-type lectin-like domain of murine receptors fused to the Fc region of human immunoglobulin $\mathrm{G1}^{4}$ (Fc-MelLec, Extended Data Fig. 1a) and used these chimaeric proteins as probes to screen for the recognition of fungi by flow cytometry. Using this approach, we identified the C-type lectin-like domain of MelLec (CLEC1A (ref. 5), Extended Data Fig. 1b), which bound A. fumigatus conidia (Fig. 1a). MelLec did not recognize other commonly occurring fungi, such as
Candida albicans yeast and filamentous cells, or Saccharomyces cerevisiae yeasts, but did recognize other melanized fungal species, including Fonsecaea pedrosoi and Cladosporium cladosporioides (Fig. 1b, Extended Data Fig. 1c, e). This indicated that the ligand recognized by MelLec was not ubiquitously found in all fungi, unlike the ligands of other antifungal CLRs such as Dectin-1 (ref. 1). Notably, the ability of MelLec to recognize A. fumigatus was restricted to conidia, and recognition was rapidly lost after conidial swelling, germination and hyphal growth (Fig. 1a, c and Extended Data Fig. 2d). The binding to conidia was visualized by immunofluorescence microscopy, which revealed a punctate staining pattern suggestive of a restricted distribution of the moiety recognized by MelLec on these spores (Fig. 1c). Conidia are covered by a hydrophobic rodlet layer, which masks underlying components of the cell wall from immune recognition ${ }^{6}$. The removal of this rodlet layer with sodium hydroxide ${ }^{6}$ led to increased and uniform binding of MelLec over the entire conidial surface (Fig. 1d). Indeed, uniform staining was obtained with rodlet-deficient conidia $(\triangle \operatorname{rod} A)$, confirming that the ligand of MelLec is partially masked by the surface hydrophobin layer (Fig. 1e). Recognition of A. fumigatus conidia by MelLec was also demonstrated in a cellular context using MelLec reporter cells ${ }^{4}$ (Extended Data Fig. 1f).

All characterized CLRs that are involved in fungal sensing act by recognition of carbohydrate components of the fungal cell wall ${ }^{1}$. Consistent with this possibility, MelLec ligand(s) were detected by enzyme-linked immunosorbent assay (ELISA) primarily in the alkali-insoluble fraction of the A. fumigatus conidial cell wall, the primary constituents of which are carbohydrates ( $\beta$-glucan, chitin and galactomannan) and melanin ${ }^{7}$ (Fig. 2a). The Fc-MelLec fusion protein was used as a probe to screen a neoglycolipid-based glycan microarray containing almost 500 structures, including oligosaccharides derived from glucans and chitin that are found in fungi ${ }^{8}$ (Supplementary Table 1). This screen did not reveal any carbohydrate ligands for MelLec; ligands were detected, however, for the C-type lectin langerin, which was used as a control (Extended Data Fig. 2). We then used Fc-MelLec to screen A. fumigatus conidia that were mutated in various relevant cell-wall biosynthetic pathways, using flow cytometry and immunofluorescence microscopy. This revealed that Fc-MelLec failed to detect $\Delta p k s P$ mutant conidia, which are deficient in the ability to synthesize heptaketide naphthopyrone (YWA1), the first intermediate of the 
a

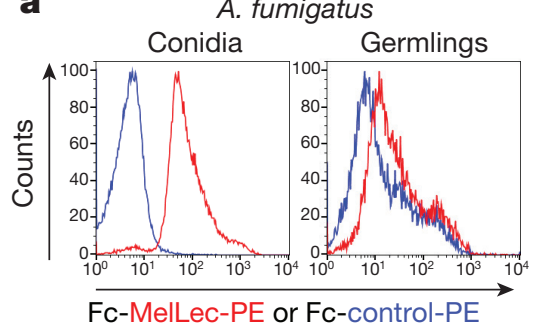

C

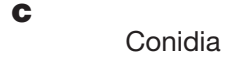

A. fumigatus
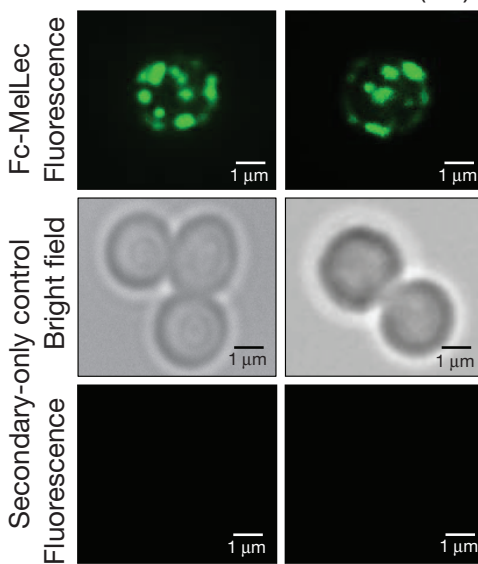

Germinating (6.5 h)

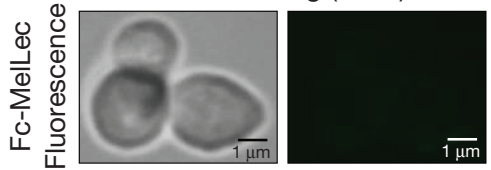

Figure 1 MelLec recognizes selected fungi. a, b, Representative histograms showing A. fumigatus conidia and germlings (cultured for $8 \mathrm{~h}$ at $\left.37^{\circ} \mathrm{C}\right)(\mathbf{a})$ and yeasts of C. albicans and S. cerevisiae, and conidia of F. pedrosoi (b) stained with Fc-MelLec or Fc-CLEC12B (ref. 24) (Fc-control) and analysed by flow cytometry. c-e, Representative light microscopy

DHN-melanin biosynthetic pathway ${ }^{2,9}$ (Fig. 2b and Extended Data Fig. 3a). We also confirmed loss of the MelLec ligand on $\triangle \operatorname{rod} A \Delta p k s P$ double mutant conidia ${ }^{10}$ — which lack both melanin and the hydrophobin layer-using flow cytometry, immunofluorescence microscopy and our MelLec reporter cells (Extended Data Figs If and 3b, c). Loss of $p k s P$ did not affect conidial recognition by Fc-Dectin-1 (Extended Data Fig. 3c, d). Moreover, we demonstrated a direct interaction of MelLec with A. fumigatus melanin ghosts (Extended Data Fig. 3e). MelLec recognized specifically DHN-melanin, as this receptor did not detect melanin synthesized by other pathways, such as those used by Cryptococcus neoformans, and mammalian B16 melanoma cells ${ }^{11}$ (Extended Data Fig. 3f). In addition to A. fumigatus, we showed that MelLec recognized other pigmented fungi that produce DHN-melanin, including F. pedrosoi and C. cladosporioides ${ }^{12}$ (Fig. 1b and Extended Data Fig. 1e).

Although its tertiary structure is unresolved ${ }^{11}$, the biosynthetic pathway of DHN-melanin is well characterized in $A$. fumigatus ${ }^{3}$. To determine at which stage MelLec ligand(s) are synthesized in the pathway, we screened mutants of $A$. fumigatus that were deficient in the enzymes required to catalyse each step, using Fc-MelLec and immunofluorescence microscopy, or ELISA. We confirmed that MelLec recognition was lost in $\Delta p k s P$ conidia; however, MelLec recognition was unaffected in mutants deficient in the enzymes required for all other stages of the DHN-melanin biosynthetic pathway (Fig. $2 \mathrm{c}$ and Extended Data Fig. 4a). Using a pre-adsorption assay, we showed that the recognition of wild-type conidia could be inhibited by pretreating Fc-MelLec with $\Delta a y g 1$ ghosts (defective in the second biosynthetic b
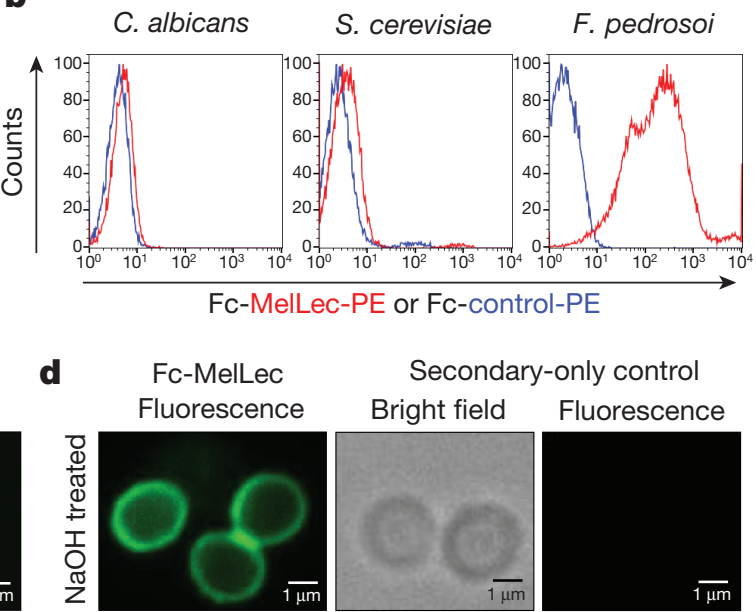

Secondary-only control

Bright field

Fluorescence
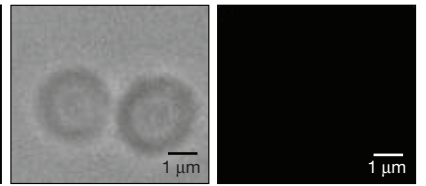

e

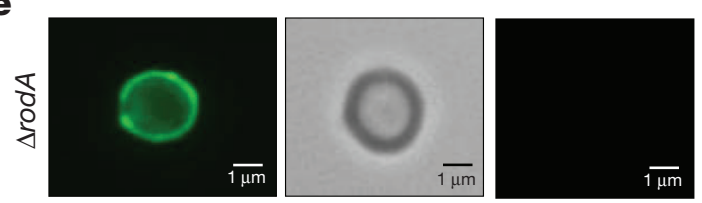

images and immunofluorescence micrographs using Fc-MelLec to detect ligands on A. fumigatus, after conidial swelling and germination over time (c), after treatment with $1 \mathrm{M} \mathrm{NaOH}(\mathbf{d})$ and on rodlet-deficient $(\triangle \operatorname{rod} A)$ conidia (e). Experiments were repeated at least three times independently, with similar results.

step, in which MelLec ligands are still present), but not ghosts of $\Delta p k s P$ (the first biosynthetic step, which lacks MelLec ligands; Extended Data Fig. 4b). We purified YWA1 from $\Delta a y g 1$ conidia $^{13}$ and demonstrated that pretreatment with this compound inhibited the ability of Fc-MelLec, but not the ability of Fc-Dectin-1, to detect $\Delta \operatorname{rodA}$ conidia or sodium hydroxide-treated wild-type conidia (Extended Data Fig. 4c, d). Moreover, using ELISA, we demonstrated a direct interaction of MelLec with purified YWA1 (Fig. 2d). This suggested that the ability of MelLec to recognize YWA1, as well as all of the other melanin biosynthetic intermediates (Fig. 2c), was due to recognition of the conserved naphthalene-diol unit, present in each of the intermediates. Indeed, we revealed a direct interaction of MelLec with 1,8-dihydronaphthalene (1,8-DHN), another upstream intermediate of the melanin biosynthetic pathway, by ELISA (Extended Data Fig. 4e). The structural isomers 1,2-DHN and 1,4-DHN, which contain the naphthalene-diol unit but are not melanin intermediates, were also recognized by MelLec (Extended Data Fig. 4f). This suggests that the position of at least one of the hydroxyls (on carbon 2, 4 or 8 ) on the naphthalene-diol unit is not important for recognition by MelLec. By contrast, naphthalene and 1-naphthol were not recognized by MelLec (Extended Data Fig. 4g), indicating that the ligand of MelLec is a naphthalene-diol.

CLRs involved in fungal recognition are predominantly expressed by myeloid cells and are detectable in most tissues ${ }^{1}$. Using reverse transcription PCR (RT-PCR), we found that MelLec was widely expressed in mice, with the highest levels of transcript detected in the lung (Extended Data Fig. 5a). To explore expression at a cellular level, 
a

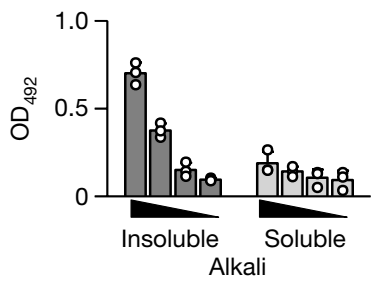

b
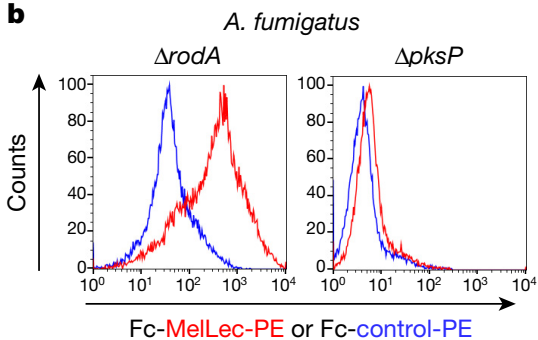

d

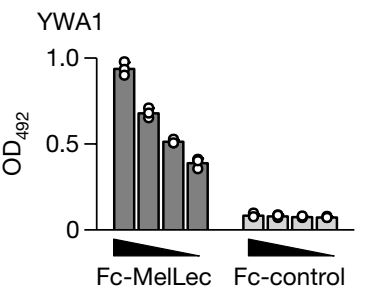

Figure 2 | MelLec recognizes DHN-melanin. a, Detection of MelLec ligands in alkali-insoluble and alkali-soluble A. fumigatus cell-wall fractions by ELISA. Values show mean \pm s.d. b, Representative histograms showing $\triangle \operatorname{rodA}$ or $\triangle p k s P$ A. fumigatus conidia, stained with FcMelLec or Fc-CLEC12B (Fc-control) and analysed by flow cytometry. c, The biosynthetic pathway of DHN-melanin (left), and representative

we generated monoclonal antibodies by immunizing rats with murine Fc-MelLec and then screening ELISA-positive hybridoma supernatants using flow cytometry on NIH3T3 cells transfected to express full-length haemagglutinin-tagged murine MelLec. In these transfected cells, we found that MelLec was expressed as a glycosylated monomer at the cell surface, which demonstrated that this CLR did not require an adaptor for surface expression ${ }^{1,14}$ (Extended Data Fig. 5b, c). Although MelLec is able to sense melanin (Fig. 2 and Extended Data Fig. 1f), the expression of this receptor on these transfected NIH3T3 cells did not confer the ability to capture A. fumigatus conidia to the cell surface (Extended Data Fig. 5d).

Two monoclonal antibodies (18E4 and 14C8) specific for MelLec were chosen for further characterization of receptor expression on mouse cells and tissues (Extended Data Fig. 5e). Surprisingly, MelLec was not expressed by any examined mouse myeloid cell population (either ex vivo or in vitro bone marrow-derived), even after microbial stimulation, nor was this receptor expressed by cells in peripheral blood, bone marrow, lymphoid tissues or on platelets ${ }^{15}$ (Extended Data Fig. $6 \mathrm{a}-\mathrm{c}$ ). Given the abundance of transcript, next we examined disaggregated lung tissue by flow cytometry, which revealed a distinct population of cells that expressed MelLec (Fig. 3a). Histological visualization of MelLec expression by immunofluorescence microscopy revealed broad punctate expression of this receptor throughout the lung tissue (Fig. 3b and Extended Data Fig. $7 \mathrm{~m}$ ). A similar punctate staining pattern was also observed in the MelLec-expressing transfected NIH3T3 fibroblasts (Extended Data Fig. 7a). Flow cytometry analysis showed that MelLec expression was restricted to non-haematopoietic $\left(\mathrm{CD}^{-} 5^{-}\right)$cells (Fig. $3 \mathrm{c}$ and Extended Data Fig. $\left.7 \mathrm{~b}\right)$. Further c

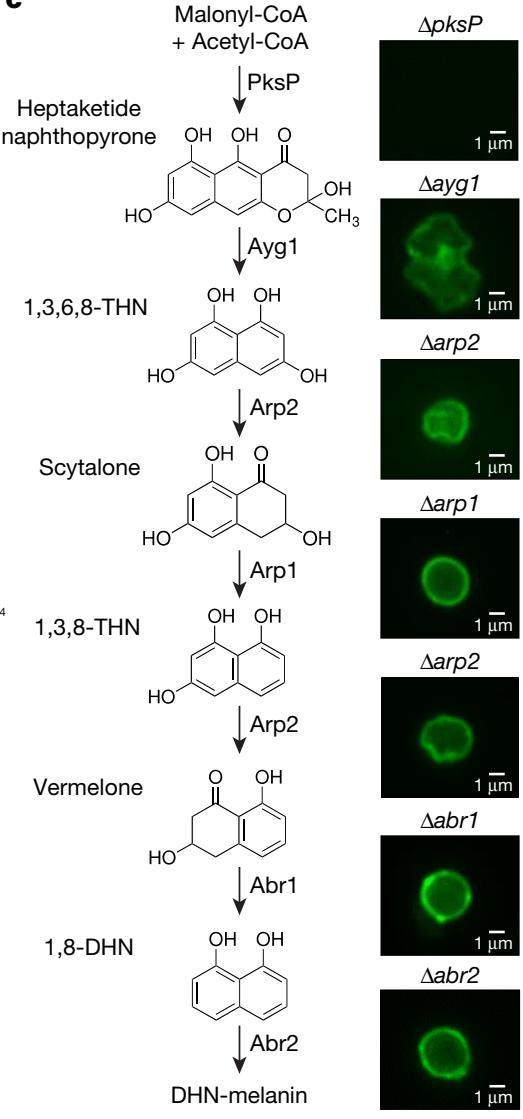

immunofluorescence micrographs obtained using Fc-MelLec to detect ligands on A. fumigatus strains deficient in the stated enzymes (right). The conidial rodlet layer was removed with $1 \mathrm{M} \mathrm{NaOH}$ before staining. d, Detection of YWA1 by Fc-MelLec and Fc-control by ELISA. Values show mean \pm s.d. Experiments were repeated at least three times independently, with similar results.

characterization of these cells revealed that MelLec was expressed by $\mathrm{CD}_{3}{ }^{+} \mathrm{EpCAM}^{-}$endothelial cells (EpCAM, epithelial cell adhesion molecule) (Fig. 3d). Expression of MelLec was also detected on CD31 ${ }^{+}$ endothelial cells in all other tissues tested, including the liver, heart, kidney and small intestine (Extended Data Fig. 7c-f). However, we also detected a unique population of $\mathrm{CD} 31^{+}$cells that co-expressed EpCAM $^{+}$, but only in the lung and the liver, and these cells also expressed MelLec (Fig. 3d and Extended Data Fig. 7c). MelLec was not expressed on $\mathrm{EpCAM}^{+}$cells in other tissues, including in the epidermis (Extended Data Fig. $7 g-j$ ).

To gain insight into the physiological functions of MelLec, we generated mice deficient in this receptor using a conventional gene-targeting vector (Extended Data Fig. 7k, 1). Exons 1-5 of the gene encoding MelLec (Clec1a) were deleted; these exons correspond to the cytoplasmic tail, transmembrane, stalk and part of the CRD region. Flow cytometry of disaggregated lung tissue, and immunofluorescence microscopy of whole lung, confirmed the lack of MelLec expression in cells from knockout mice (Fig. 3b and Extended Data Fig. 7m, n). The MelLec-knockout mice were viable, had no gross abnormalities and had normal peripheral leucocyte counts (Supplementary Table 2). Intratracheal (i.t.) challenge of immunocompetent MelLec-knockout mice with wild type $A$. fumigatus conidia revealed no alterations in survival or in other physiological parameters, including weight (Extended Data Fig. 8a). However, a significantly reduced influx of neutrophils into the lungs of MelLec-knockout mice could be detected shortly $(4 \mathrm{~h})$ after i.t. challenge (Extended Data Fig. 8b-d), before the conidia had germinated ${ }^{16}$. This alteration in cellular recruitment in the MelLec-knockout mice was associated with alterations in selected 

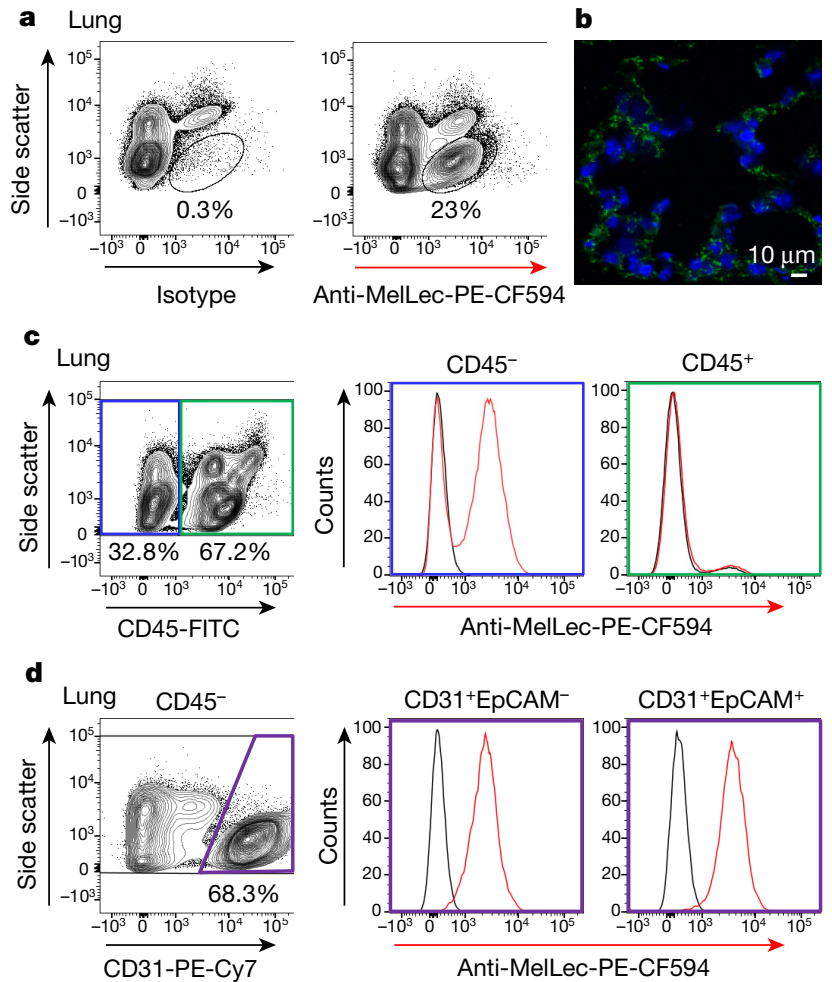

Figure 3 MelLec is expressed on non-myeloid cells in mouse. a, Analysis of disaggregated lung tissue by flow cytometry with antiMelLec antibody. b. Immunofluorescence micrographs of lung tissue stained with anti-MelLec antibody (green). Nuclei are stained with 4',6-diamidino-2-phenylindole (DAPI) (blue). c, d, Flow-cytometric analysis of MelLec expression on live CD $45^{+}$and CD $45^{-}$cells (c), and $\mathrm{CD}_{4} 5^{-} \mathrm{CD} 31^{+} \mathrm{EpCAM}^{-}$, and $\mathrm{CD} 45^{-} \mathrm{CD} 31^{+} \mathrm{EpCAM}^{+}$cells $(\mathbf{d})$ in the lung. Experiments were repeated at least three times independently, with similar results.

neutrophil-related cytokines, including KC (CXCL1) and granulocyte macrophage colony-stimulating factor (Extended Data Fig. 8e). A similar alteration in neutrophil recruitment was also observed in the MelLec-knockout mice after i.t. challenge with melanin ghosts of $A$. fumigatus (Extended Data Fig. 8f). There were no alterations in other pulmonary myeloid populations after conidial challenge, and by $24 \mathrm{~h}$ after the challenge the difference in neutrophil influx was no longer apparent (Extended Data Fig. 8g, h). There was also no change in the expression of MelLec during infection with A. fumigatus (Extended Data Fig. 8i). Notably, the defect in early neutrophil-recruitment in the MelLec-knockout mice was lost upon i.t. challenge with $\Delta p k s P$ conidia, which lack melanin (Extended Data Fig. 8j, k).

We next examined the role of MelLec during infections in corticosteroid-treated mice, to model the effects of immunosuppression ${ }^{17}$. Under these conditions, loss of MelLec did not significantly alter the susceptibility to infection (Extended Data Fig. 9a). However, when we intravenously (i.v.) infected immunocompetent MelLec-knockout mice with $A$. fumigatus conidia ${ }^{17}$, we observed substantially increased susceptibility in these mice compared to the wild type (Fig. 4a). This increased susceptibility was associated with increased fungal burdens in several tissues, including the brain (Fig. $4 \mathrm{~b}$ and Extended Data Fig. 9b-d), as well as alterations in inflammatory responses (Fig. 4c). IL-17 responses were unaffected ${ }^{18}$. Consistent with a role in melanin recognition, there was no difference in susceptibility or fungal burden between wild-type and MelLec-knockout mice after systemic infection with $\Delta p k s P$ conidia (Fig. $4 \mathrm{~d}$ and Extended Data Fig. 9e).

Our mouse data suggested that MelLec has a key role in the immunity to disseminated infections with Aspergillus. Therefore, we next explored the role of this receptor in humans. MelLec has been a

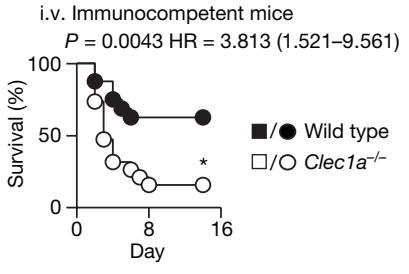

b

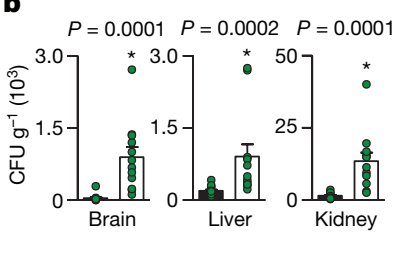

C Brain, day 4

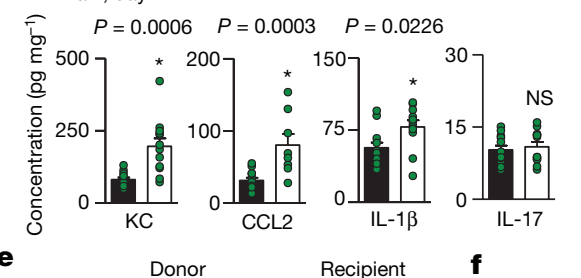

d i.v. Immunocompetent mice

$\Delta p k s P$

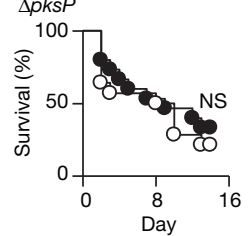

Donor $\begin{gathered}\text { Dor } \\ P=0.003 \\ 39 \pm 8.3 \% \quad \text { * }\end{gathered}$
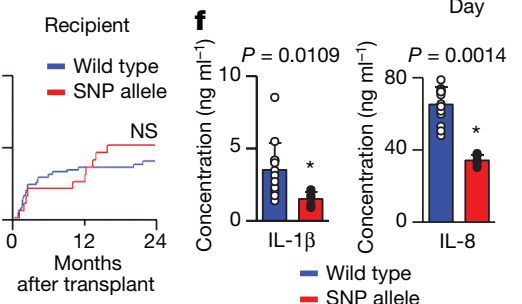

Figure $4 \mid$ MelLec is required to prevent disseminated infection in mice and humans. a, Survival of mice after i.v. infection with $10^{6} \mathrm{~A}$. fumigatus conidia ( $n=16$ mice per group). Pooled data from two independent experiments, analysed by log-rank test. b, c, Tissue fungal burdens (b) and brain cytokine levels (c) of mice four days after i.v. infection with $10^{6}$ A. fumigatus conidia ( $n=12$ mice per group). CFU, colony-forming unit. Values shown are mean \pm s.e.m. of pooled data from two independent experiments, analysed by two-sided Mann-Whitney $U$ test. Green circles indicate individual data points in all cases. d, Survival of mice after i.v. infection with $10^{6} \Delta p k s P$ A. fumigatus conidia ( $n=15$ mice per group). Pooled data from two independent experiments, analysed by log-rank test. e, Cumulative incidence analysis of invasive aspergillosis after transplantation according to donor (wild-type, $n=238$ individuals; SNP allele, $n=72$ individuals) or recipient (wild-type, $n=228$ individuals; SNP allele, $n=80$ individuals) CLEC1A rs2306894 genotypes, analysed by two-sided Gray's test. f, Cytokine production in monocyte-derived macrophages, after stimulation with A. fumigatus conidia (wild-type, $n=14$ individuals; SNP allele, $n=5$ individuals). Values shown are mean \pm s.d., analysed by two-sided Mann-Whitney $U$ test. $* P \leq 0.05$; NS, not significant; HR, hazard ratio.

previously detected in humans on endothelial cells ${ }^{19,20}$ but also on myeloid cells ${ }^{5,18-20}$, and we found that a human MelLec Fc fusion protein $(\mathrm{Fc}-\mathrm{hMelLec})$ recognizes DHN-melanized conidia (Extended Data Fig. 10a). A common missense single nucleotide polymorphism (SNP) within the coding region of CLEC1A (rs2306894, global minor allele frequency $=0.3295$ ) results in an amino acid change (Gly26Ala) in the cytoplasmic tail of human MelLec. Notably, we found a highly significant association between this SNP and the risk of aspergillosis in stem-cell transplant recipients (Fig. 4e). This increased risk occurred when the variant was carried by the donor, but not when it was carried by the recipient. This suggests that, in humans, the protective functions of MelLec are primarily mediated by myeloid cells. In our mouse model, there was no difference in resistance to infection upon adoptive transfer of MelLec-deficient bone marrow into irradiated wild-type recipients (Extended Data Fig. 10b).

To demonstrate the effect of the SNP in CLEC1A and subsequent amino acid substitution in MelLec on the function of myeloid cells, we analysed the responses of monocyte-derived macrophages isolated from healthy genotyped donors. We found that macrophages from the individuals carrying this SNP produced significantly less IL-1 $\beta$ and IL-8 after in vitro stimulation with $A$. fumigatus conidia compared to controls (Fig. 4f), whereas there was no difference in response upon stimulation with lipopolysaccharide (Extended Data Fig. 10c). We verified 
the inflammatory defect caused by this SNP in peripheral blood mononuclear cells isolated from an independent cohort (Extended Data Fig. 10d). Moreover, using transduced RAW264.7 macrophages, we demonstrated directly that this SNP results in an inflammatory defect upon stimulation with melanin-containing conidia (Extended Data Fig. 10e).

Melanin is considered a fungal virulence factor, providing protection against reactive oxygen species and inhibiting host-cell phagocytosis, cytokine production and apoptosis $\mathrm{s}^{3,10,21}$. Here we show that fungal DHN-melanin is also sensed by the host, through a melanin-sensing C-type lectin receptor (MelLec) that has a crucial role in the control of systemic $A$. fumigatus infection in both mice and humans. However, the data presented here, as well as early studies in rats ${ }^{18,22}$, show that the cellular expression of this receptor differs between species. Critically, we define a polymorphism of this receptor that, when present in donor cells, increases the susceptibility of stem-cell transplant recipients to disseminated aspergillosis. Our data therefore suggest that identifying donors carrying this SNP could help considerably to reduce the incidence of this disease in transplant recipients. It is likely that MelLec will have an important role in immunity to other melanized fungi and black yeasts $^{23}$, especially those that cause phaeohyphomycosis, mycetoma and chromoblastomycosis.

Online Content Methods, along with any additional Extended Data display items and Source Data, are available in the online version of the paper; references unique to these sections appear only in the online paper.

Received 3 October 2016; accepted 6 February 2018.

Published online 28 February 2018.

1. Hardison, S. E. \& Brown, G. D. C-type lectin receptors orchestrate antifungal immunity. Nat. Immunol. 13, 817-822 (2012).

2. Nosanchuk, J. D. \& Casadevall, A. The contribution of melanin to microbial pathogenesis. Cell. Microbiol. 5, 203-223 (2003).

3. Heinekamp, T. et al. Aspergillus fumigatus melanins: interference with the host endocytosis pathway and impact on virulence. Front. Microbiol. 3, 440 (2013).

4. Pyż, E. \& Brown, G. D. Screening for ligands of C-type lectin-like receptors. Methods Mol. Biol. 748, 1-19 (2011).

5. Colonna, M., Samaridis, J. \& Angman, L. Molecular characterization of two novel $\mathrm{C}$-type lectin-like receptors, one of which is selectively expressed in human dendritic cells. Eur. J. Immunol. 30, 697-704 (2000).

6. Aimanianda, V. et al. Surface hydrophobin prevents immune recognition of airborne fungal spores. Nature 460, 1117-1121 (2009).

7. Latgé, J. P. \& Beauvais, A. Functional duality of the cell wall. Curr. Opin. Microbiol. 20, 111-117 (2014).

8. Palma, A. S. et al. Ligands for the $\beta$-glucan receptor, Dectin-1, assigned using "designer" microarrays of oligosaccharide probes (neoglycolipids) generated from glucan polysaccharides. J. Biol. Chem. 281, 5771-5779 (2006).

9. Jahn, B. et al. Isolation and characterization of a pigmentless-conidium mutant of Aspergillus fumigatus with altered conidial surface and reduced virulence. Infect. Immun. 65, 5110-5117 (1997).

10. Akoumianaki, T. et al. Aspergillus cell wall melanin blocks LC3-associated phagocytosis to promote pathogenicity. Cell Host Microbe 19, 79-90 (2016).

11. Nosanchuk, J. D., Stark, R. E. \& Casadevall, A. Fungal melanin: what do we know about structure? Front. Microbiol. 6, 1463 (2015).

12. Llorente, C. et al. Cladosporium cladosporioides LPSC 1088 produces the 1,8-dihydroxynaphthalene-melanin-like compound and carries a putative pks gene. Mycopathologia 174, 397-408 (2012).

13. Tsai, H. F. et al. Pentaketide melanin biosynthesis in Aspergillus fumigatus requires chain-length shortening of a heptaketide precursor. J. Biol. Chem. 276, 29292-29298 (2001).
14. Sancho, D. \& Reis e Sousa, C. Signaling by myeloid C-type lectin receptors in immunity and homeostasis. Annu. Rev. Immunol. 30, 491-529 (2012).

15. Rambach, G. et al. Identification of Aspergillus fumigatus surface components that mediate interaction of conidia and hyphae with human platelets. J. Infect. Dis. 212, 1140-1149 (2015).

16. Faro-Trindade, I. et al. Characterisation of innate fungal recognition in the lung. PLOS ONE 7, e35675 (2012).

17. Clemons, K. V. \& Stevens, D. A. The contribution of animal models of aspergillosis to understanding pathogenesis, therapy and virulence. Med. Mycol. 43 (Suppl. 1), 101-110 (2005)

18. Lopez Robles, M. D. et al. Cell-surface C-type lectin-like receptor CLEC-1 dampens dendritic cell activation and downstream Th17 responses. Blood Adv. 1, 557-568 (2017)

19. Sobanov, Y. et al. A novel cluster of lectin-like receptor genes expressed in monocytic, dendritic and endothelial cells maps close to the NK receptor genes in the human NK gene complex. Eur. J. Immunol. 31, 3493-3503 (2001).

20. Sattler, S. et al. The human C-type lectin-like receptor CLEC-1 is upregulated by TGF- $\beta$ and primarily localized in the endoplasmic membrane compartment. Scand. J. Immunol. 75, 282-292 (2012).

21. Chai, L. Y. et al. Aspergillus fumigatus conidial melanin modulates host cytokine response. Immunobiology 215, 915-920 (2010).

22. Thebault, P. et al. The C-type lectin-like receptor CLEC-1, expressed by myeloid cells and endothelial cells, is up-regulated by immunoregulatory mediators and moderates T cell activation. J. Immunol. 183, 3099-3108 (2009).

23. Seyedmousavi, S. et al. Black yeasts and their filamentous relatives: principles of pathogenesis and host defense. Clin. Microbiol. Rev. 27, 527-542 (2014).

24. Hoffmann, S. C. et al. Identification of CLEC12B, an inhibitory receptor on myeloid cells. J. Biol. Chem. 282, 22370-22375 (2007).

Supplementary Information is available in the online version of the paper.

Acknowledgements We thank the staff of the University of Aberdeen animal facility for their support and care for our animals, C. G. Park for providing recombinant langerin, and S. Filler and R. Cramer for advice. Funding was provided by the Wellcome Trust (102705, 097377, 093378, 099197, 108430, 101873), the Medical Research Council Centre for Medical Mycology and the University of Aberdeen (MR/N006364/1). K.J.K.-C is supported by the intramural program of the National Institute of Allergy and Infectious Diseases, National Institutes of Health; V.A. by an ANR-DST COMASPIN grant (ANR-13ISV3-0004); B.H. by German Science Foundation (www.dfg.de) grant no. HE 7565/1-1; J.-P.L., I.V. and V.A. by the ANR and FRM DEQ2015-331722; A.C. by the Northern Portugal Regional Operational Programme (NORTE 2020), under the Portugal 2020 Partnership Agreement, through the European Regional Development Fund (FEDER) (NORTE-01-0145-FEDER-000013), and by the Fundação para a Ciência e Tecnologia (FCT) (IF/00735/2014 and SFRH/ BPD/96176/2013).

Author Contributions G.D.B., J.-P.L. and J.A.W. conceived and designed the study and guided the interpretation of the results. A.E.C., M.H.T.S. and V.A. performed the majority of the experiments and data analysis. S.B., D.M.R., P.A., S.E.H., I.M.D., B.K., A.P., J.A.W., C.C., M.d.G.T.S., C.A.W., R.Y. and B.H. conducted experiments and data analysis. Y.L. and T.F. performed the glycan microarray experiments and data analysis. M.J., M.G.N., F.L.v.d.V., J.F.L., A.Cam. and A.Car. provided the human patient data and analysis. A.A.B., K.J.K.-C., I.V., M.P., C.Z., M.Z. and N.A.R.G. provided critical conceptual input and reagents. G.D.B. drafted the manuscript. All authors discussed the results, edited and approved the draft and final versions of the manuscript.

Author Information Reprints and permissions information is available at www.nature.com/reprints. The authors declare no competing interests. Readers are welcome to comment on the online version of the paper. Publisher's note: Springer Nature remains neutral with regard to jurisdictional claims in published maps and institutional affiliations. Correspondence and requests for materials should be addressed to G.D.B. (gordon.brown@abdn.ac.uk).

Reviewer Information Nature thanks A. Casadevall, C. Reis e Sousa and the other anonymous reviewer(s) for their contribution to the peer review of this work. 


\section{METHODS}

Mice and fungal strains. $\mathrm{C} 57 \mathrm{BL} / 6$ and $\mathrm{Clec} \mathrm{a}^{-1-}$ mice (8-12 weeks old) were obtained from the specific pathogen-free facility at the University of Aberdeen. Animal experiments were performed using age-matched female mice and conformed to the animal care and welfare protocols approved by the UK Home Office (project license 70/8073) in compliance with all relevant local ethical regulations. Clec $1 a^{-1-}$ mice were generated commercially (TaconicArtemis) by conventional gene targeting in C57BL/6 embryonic stem cells, as detailed in Extended Data Fig. 7. Sample sizes of at least five animals per group were chosen as this would allow the detection of a $25 \%$ difference in the mean between experimental and control groups with a probability of greater than $95 \%(P<0.05)$, assuming a standard deviation of around $15 \%$ and a minimum power value of 0.8 . Mice were randomly assigned to experimental or control groups, co-housed, and experiments were not blinded.

A. fumigatus isolate 13073 (American Type Culture Collection (ATCC)) and a clinical isolate CBS 144-89 (ref. 25) were used as wild-type strains. Melanin mutant strains ( $\triangle$ alb1 (pksP1), $\Delta$ ayg1, $\Delta a b r 1, \Delta a b r 2, \Delta \operatorname{arp} 1$ and $\Delta a r p 2$ in the B5233 wild-type background) were generated previously ${ }^{26} . \Delta \operatorname{rod} A$ and $\triangle p k s P \Delta \operatorname{rodA}$ deletion mutants were generated as described ${ }^{9,10,25}$. Wild-type CBS110.46 (ref. 27) and CBS386.75 (ref. 27) strains with white conidia were also used, as indicated. All strains were maintained on $2 \%(\mathrm{w} / \mathrm{v})$ malt-agar slants or potato dextrose agar in culture flasks; conidia were collected and washed before use. Swollen and/or germinating morphotypes were obtained upon incubating conidia in Sabouraud liquid culture medium at $37^{\circ} \mathrm{C}$ for different time intervals, as indicated.

Fc-MelLec production and immunolabelling. Soluble chimaeric proteins containing the stalk and C-type lectin-like domain of mouse and human MelLec fused to the mutated Fc portion of human immunoglobulin G1 (IgG1) were generated essentially as described previously ${ }^{28}$. In brief, the relevant portions of the MelLec encoding genes were amplified by PCR (mouse primers, GAATTCCTCGAGCTGGAGCTCTCCAGGTAC and AAGCTTTCTA CCAGCTGTCTAAT; human primers, GGGATCCACTACTACCAGCTCTCC and TGGATATCTGTCACCTTCGCCTAATGTTTC), and cloned into the pSecTag2 expression vector (Invitrogen Life Technologies) containing the mutated form of human IgG1 (ref. 29). Sequenced constructs were transfected into HEK293T cells using Fugene 6 (Promega), as per the manufacturer's instructions. Fusion proteins were purified from conditioned supernatants by chromatography on protein A Sepharose and dialysed against PBS. Fc-Dectin-1 (ref. 28) and Fc-CLEC12B (ref. 24) were generated similarly and used as controls.

For flow cytometry, fungi were incubated in $1-3 \%(\mathrm{w} / \mathrm{v})$ bovine serum albumin (BSA) in PBS and Fc-proteins were added to a final concentration of $5 \mu \mathrm{g} \mathrm{ml}^{-1}$. Following incubation at $4^{\circ} \mathrm{C}$, fungal particles were washed in fluorescenceactivated cell sorting (FACS) buffer (0.5\% (w/v) BSA and $2 \mathrm{mM}$ EDTA in PBS), and bound Fc-proteins detected with allophycocyanin (APC) or phycoerythrin (PE)-conjugated donkey anti-human antibody (Jackson ImmunoResearch), fixed in $1 \%(\mathrm{v} / \mathrm{v})$ formaldehyde, and analysed.

For microscopy, paraformaldehyde-fixed conidia were taken for immunolabelling ${ }^{6}$. To obtain $\mathrm{NaOH}$-treated conidia, $1 \mathrm{M} \mathrm{NaOH}$ was added to conidia and heated in a boiling water bath for $1 \mathrm{~h}$, followed by centrifugation to collect conidia and extensive washing. Melanin ghosts were isolated from conidia (B5233 as well as melanin mutant strains) by harsh chemical treatments that degrade other cellular components and result in hollow spherical-shaped melanin shells ('melanin ghosts'), as described previously ${ }^{30}$. For immunostaining, fungal particles were incubated with $5 \mu \mathrm{g} \mathrm{ml}^{-1} \mathrm{Fc}$-MelLec in PBS containing $1 \%(\mathrm{w} / \mathrm{v})$ BSA for $1 \mathrm{~h}$. After washing, bound Fc-MelLec was detected with fluorescein isothiocyanate (FITC)-labelled goat anti-human Fc-specific IgG (Sigma) in PBS containing $1 \%(\mathrm{w} / \mathrm{v}) \mathrm{BSA}$ and washed with PBS-Tween. Conidia incubated with FITC-labelled anti-human Fc-specific IgG only were used as a negative control (data not shown). Labelled conidia were observed by fluorescence microscopy (Leica DMLB).

In some experiments, Fc-proteins were pretreated with either ghosts of melanin mutant strains, as indicated, or YWA1, which was isolated from AYG1 deletion mutant conidia as described previously ${ }^{13}$.

Monoclonal antibody production. The generation of monoclonal antibodies to MelLec was performed essentially as described previously ${ }^{31}$. In brief, Sprague Dawley rats were immunized with Fc-MelLec in Freund's complete adjuvant. After a final intraperitoneal boost, without adjuvant, rat splenocytes were collected and fused with Y3 myeloma cells, as described ${ }^{32}$. Hybridoma supernatants were screened by ELISA and positives were then tested by immunohistochemistry and flow cytometry, as described below, against Fc-MelLec as well as MelLectransduced NIH3T3 fibroblasts. Two monoclonal antibodies (14C8 and 18E4; both IgG1) were selected for further use. Where required, they were biotinylated with Sulfo-NHS-LC Biotin (Pierce), as described by the manufacturer.
Cell culture and growth conditions. Cells were maintained at $37^{\circ} \mathrm{C}$ and $5 \% \mathrm{CO}_{2}$ in DMEM or RPMI medium supplemented with $10 \%$ heat-inactivated fetal calf serum, 100 units per $\mathrm{ml}$ penicillin, $0.1 \mathrm{mg} \mathrm{ml}^{-1}$ streptomycin, and $2 \mathrm{mM} \mathrm{L}$-glutamine. Phoenix ecotropic packaging cells (Plat-E) were maintained with the addition of $1 \mu \mathrm{g} \mathrm{ml}^{-1}$ puromycin and $10 \mu \mathrm{g} \mathrm{ml}^{-1}$ blasticidin (Life Technologies, Inc.)

NIH3T3 fibroblasts stably expressing full-length murine or human MelLec were generated essentially as described for Dectin-1 (ref. 33). In brief, haemagglutinintagged MelLec was generated through PCR amplification (mouse primers, GGA TCCACCATGCAGGCCAAATACAGCA and CTCGAGCTACTGGAGCT CTCCAGGTAC; human primers, AAAGGATCCACCATGCAGGCCAAGT ACAGCAGCAC and AGCGTAATCCGGAACATCGTATGGGTACTCGAG) and subcloning into $\mathrm{pFb}$-neo (Stratagene). Constructs were transfected into Plat-E retroviral packaging cell lines using Fugene 6 and retrovirus-containing supernatants were used to transduce NIH3T3 fibroblasts in the presence of polybrene (Sigma). Stably transfected cells were selected using $600 \mu \mathrm{g} \mathrm{ml}^{-1} \mathrm{G} 418$ (ThermoFisher Inc.). The expression of the receptor was confirmed by flow cytometry and western blotting, using the anti-haemagglutinin antibody (Covance). In some experiments, NIH3T3 cells expressing murine CLEC12A (ref. 31) were used as controls.

ELISA assays. Alkali-insoluble and soluble fungal cell wall fractions were obtained as described previously ${ }^{34}$. For the ELISA, wells were coated overnight with $20-200 \mu \mathrm{g} \mathrm{ml}^{-1}$ of the cell wall fractions, melanin ghosts (in-house generated), purified YWA1 (in-house generated), 1,8-DHN, 1,2-DHN, 1,4-DHN, naphthalene, 1-naphthol (all from Sigma) in $50 \mathrm{mM}$ carbonate buffer $\mathrm{pH}$ 9.6, and then blocked with $1 \%$ BSA in PBS. Fc-MelLec $\left(5 \mu \mathrm{g} \mathrm{ml}^{-1}\right)$ was added to the wells and incubated for $1 \mathrm{~h}$ at room temperature. After washing with PBS-Tween-20 (0.5\% (v/v)), peroxidase-conjugated human Fc-specific IgG (Sigma) was added to the wells and incubated for $1 \mathrm{~h}$ at room temperature. After further washing, quantification of Fc-MelLec binding was detected using $o$-phenylenediamine (Sigma) and $\mathrm{H}_{2} \mathrm{O}_{2}$ detection system (Merck). The reaction was stopped using $4 \%$ (v/v) $\mathrm{H}_{2} \mathrm{SO}_{4}$ and optical densities were measured at $492 \mathrm{~nm}$.

Expression analysis. Peripheral blood leucocytes, resident and thioglycolateelicited inflammatory peritoneal cells, alveolar macrophages and bone marrow cells were isolated essentially as described previously ${ }^{35}$. For platelets, peripheral blood was collected in $3.8 \%(\mathrm{w} / \mathrm{v})$ sodium citrate buffer and centrifuged at $200 \mathrm{~g}$ at $25^{\circ} \mathrm{C}$. The supernatant, containing platelet-rich plasma, was used for subsequent analysis. Bone marrow-derived macrophages or dendritic cells, generated using L929 conditioned medium or $20 \mathrm{ng} \mathrm{ml}^{-1}$ granulocyte-macrophage colonystimulating factor (R\&D Systems), respectively, were prepared as described ${ }^{31}$. In some experiments, cells were stimulated with $100 \mathrm{ng} \mathrm{ml}^{-1}$ lipopolysaccharide from Escherichia coli (Sigma).

Tissues isolated from mice were cut into small pieces and incubated for $30 \mathrm{~min}$ at $37^{\circ} \mathrm{C}$ with Liberase (Roche) and DNase (Roche) in RPMI (Gibco), except for the small intestine which was incubated with Collagenase VIII (Sigma-Aldrich). Cells were disaggregated using the gentleMACS Dissociator (Miltenyi), strained through $70-\mu \mathrm{m}$ nylon cell strainers (Fisher Scientific) and collected by centrifugation. Red blood cells were removed using Pharm Lyse (BD Biosciences).

For flow cytometry, isolated cells were washed in FACS wash (PBS with $0.5 \%$ $(\mathrm{w} / \mathrm{v}) \mathrm{BSA}$ and 5-10 mM EDTA) containing anti-CD16/CD32 (Clone 2.4G2, prepared in house). The following antibodies (all from BD Biosciences, eBioscience or Abcam) were used for FACS analysis of cell surface antigen expression following standard methodology: anti-MelLec-Biotin (described above), StreptavidinPE-CF594 or Streptavidin-APC, anti-CD45.2-FITC (clone 104), biotinylated anti-CD61 (clone 2C9.G2), anti-CD326-APC (EpCAM; clone G8.8), anti-CD31PE-Cyanine7 (PECAM-1; clone 390), anti-Ly6G-APC (clone 1A8), anti-CD11bPE-Cy7 (clone M1/70), CD11c-PerCP-Cy5.5 (clone HL3), anti-Siglec-F-BV421 (clone E50-2440), anti-CD45-PE-Cy7 (clone 30-F11), anti-CD11b-PerCP-Cy5.5 (clone M1/70), anti-CD11c-BV421 (clone HL3), anti-Siglec-F-PE (clone E502440) and anti-F4/80-AF700 (clone CL:A3-1) and isotype control AFRC MAC 49 (ECACC 85060404; isotype for anti-MelLec). Cell viability was detected using the fixable viability dye eFluor-780 (eBioscience) in PBS, and the cells were fixed with $1 \%$ formaldehyde $(\mathrm{v} / \mathrm{v})$ before acquisition on a LSRII, LSR Fortessa or FACS Calibur (Becton Dickinson). Data were analysed using FlowJo. All contour plots were constructed using $2 \%$ probability contouring with outliers.

For immunofluorescence microscopy, $6 \mu \mathrm{m}$ alcohol-fixed frozen lung sections were treated with Liberase (Roche) for $4 \mathrm{~min}$ at room temperature. Sections were blocked with $2 \%(\mathrm{v} / \mathrm{v})$ normal goat serum, and incubated with anti-MelLec or isotype control for $1 \mathrm{~h}$ followed by AlexaFluor 488 goat anti-rat (Invitrogen) for 30 min. Vectashield with DAPI or propidium iodide (Vector Laboratories Inc.) was used as a mountant for fluorescence, and slides were visualized using a Zeiss LSM 700 confocal microscope. Visualization of transfected NIH3T3 cells was performed similarly, except the cells were fixed in $4 \%(\mathrm{v} / \mathrm{v})$ paraformaldehyde before analysis. 
Detection of MelLec in complementary DNA (cDNA) (Multiple Tissue Panels, Clontech) was performed using the Titanium Taq PCR kit (Clontech) with the following primers: CAGAGCCCAGGCACTCAGAGAATG and TGCGGGAGAGCCCTGTCCAAT. Expression of G3PDH was detected as described previously ${ }^{36}$.

Mouse infection models. For pulmonary infections, $10^{5}$ (corticosteroid model) or $10^{7}$ A. fumigatus ATCC 13073 conidia were administered to the caudal oropharynx of anaesthetized mice. In some experiments, mice were administered with $0.6 \mathrm{mg}$ of the corticosteroid, triamcinolone acetonide ${ }^{37}$ (Bristol-Myers Squibb), on days $-1,1$ and 3 , relative to infection on day 0 . For systemic infections, $10^{6} \mathrm{~A}$. fumigatus ATCC 13073 conidia were administered to the lateral tail vein of mice. Mice were killed when they had lost $30 \%$ body weight or had become moribund. Pulmonary cellular inflammation was assessed by flow cytometry after total lung enzymatic digest, as described above, or in bronchoalveolar lavage samples, isolated with PBS containing $5 \mathrm{mM}$ EDTA (Gibco). Organs were homogenized in PBS and used for the determination of fungal burdens and levels of inflammatory cytokines. Fungal burdens were determined by serial dilution onto potato dextrose agar plates and normalized to organ weights. Cytokines were measured by ELISA (R\&D DuoSet), as described by the manufacturer, and normalized to protein concentration. Bone marrow chimaeric mice were generated as described previously ${ }^{38}$.

Glycan microarray analyses. Microarray analyses were carried out using the neoglycolipid-based microarray system ${ }^{39}$. Details of the glycan probe library, the generation of the microarrays, imaging and data analysis are in the supplementary glycan microarray document (Supplementary Table 3) in accordance with the MIRAGE (minimum information required for a glycomics experiment) guidelines for reporting glycan microarray-based data. The microarray contained 496 lipidlinked glycan probes (Supplementary Table 1). Microarray analysis of the soluble Fc-MelLec was performed essentially as described ${ }^{8}$. In brief, after blocking arrayed slides with $0.04 \%$ (v/v) Blocker Casein (Pierce), 1\% (w/v) BSA (Sigma A8577) in HEPES-buffered saline ( $5 \mathrm{mM}$ HEPES, pH 7.4, $150 \mathrm{mM} \mathrm{NaCl}, 5 \mathrm{mM} \mathrm{CaCl}_{2}$ ), mouse Fc-MelLec was precomplexed with biotinylated anti-human IgG (Vector) at a 1:3 ratio $(\mathrm{w} / \mathrm{w})$ before application onto the slides at a final concentration of $10 \mu \mathrm{g}$ $\mathrm{ml}^{-1}$. Flag-tagged human langerin was included as a positive control. The protein and rat anti-Flag antibody mAb L5 were provided by C. G. Park. For analysis, the Flag-tagged langerin was precomplexed with the rat anti-Flag at a 1:3 ratio $(\mathrm{w} / \mathrm{w})$ and applied onto the slides at a final concentration of $5 \mu \mathrm{g} \mathrm{ml}^{-1}$, followed by biotinylated anti-rat IgG (Pierce) $\left(5 \mu \mathrm{g} \mathrm{ml}^{-1}\right)$. To detect binding, AlexaFluor-647labelled streptavidin from Molecular Probes was used at $1 \mu \mathrm{g} \mathrm{ml}^{-1}$. Data analysis and presentation was performed with dedicated glycan microarray software ${ }^{40}$.

Human studies. A total of 310 haematologic patients undergoing allogeneic haematopoietic stem cell transplantation at the Hospital of Santa Maria, Lisbon and Instituto Português de Oncologia (IPO), Porto, between 2009 and 2014 were enrolled in the study. The cases of invasive aspergillosis were identified and classified as 'probable' or 'proven' according to the revised standard criteria from the European Organization for Research and Treatment of Cancer/Mycology Study Group (EORTC/MSG) ${ }^{41}$. Exclusion criteria included diagnosis of 'possible' invasive aspergillosis, infection with invasive moulds other than Aspergillus spp. or history of pre-transplant mould infection. Study approval was obtained from the institutional review boards (SECVS-125/2014, HSM-632/14 and CES.26/015) and from the National Data Protection Commission (CNPD, 1950/2015) and was in compliance with all local relevant ethical regulations.

Genomic DNA was isolated from whole blood of recipients and donors (before transplantation) using the QIAcube automated system (Qiagen) at the regional centres of the Instituto Português do Sangue e Transplantação (Portugal). Genotyping of the nonsynonymous rs2306894 SNP in the CLEC1A gene was performed using KASPar assays (LGC Genomics) according to the manufacturer's instructions in an Applied Biosystems 7500 Fast real-time PCR system (Thermo Fisher). Genotyping sets included randomly selected replicates of previously typed samples, and agreement between original and duplicate samples was $\geq 99 \%$.

Peripheral blood mononuclear cells from healthy genotyped donors were enriched from buffy coats using Histopaque-1077 (Sigma-Aldrich) and contaminating erythrocytes were removed using Red Blood Cell Lysis Buffer (SigmaAldrich). Participants gave written informed consent before blood collection. Monocytes were isolated by positive selection using magnetically labelled $\mathrm{CD} 14^{+}$ MicroBeads (Miltenyi Biotec) on a MiniMACS separator and seeded at $10^{6}$ cells per $\mathrm{ml}$ in 24-well plates for 7 days in RPMI-1640 medium supplemented with $10 \%(\mathrm{v} / \mathrm{v})$ human serum and $20 \mathrm{ng} \mathrm{ml}^{-1}$ recombinant human granulocyte macrophage colony-stimulating factor (GM-CSF, Gibco). Acquisition of macrophage morphology was confirmed by phase contrast microscopy (Axiovert 135, Zeiss). For infection, macrophages were washed and then infected with live conidia of A. fumigatus strain A1163 at a ratio of $1: 10$ (cells:fungus) for $20 \mathrm{~h}$ at $37^{\circ} \mathrm{C}$ and $5 \% \mathrm{CO}_{2}$. Cytokines in supernatants were detected using DuoSet ELISA systems
(BioLegend), according to the manufacturer's instructions. At least two technical replicates were performed for each donor.

For the independent cohort, genomic DNA was isolated from EDTA venous blood of healthy Dutch volunteers using the Gentra Pure Gene Blood kit (Qiagen) and genotyped for CLEC1A polymorphisms using the Illumina Immunochip SNP array platform, described previously ${ }^{42}$. Participants gave written informed consent before blood collection. As the CLEC1A SNP of interest (exonic rs2306894) was not represented on the genotyping platform, two intronic polymorphisms (rs7972187 and rs3825300) were used as markers. Linkage analysis revealed complete linkage disequilibrium of these two polymorphisms with the SNP of interest ${ }^{43}\left(R^{2}=1\right)$. A total of $10^{5}$ peripheral blood mononuclear cells isolated from genotyped donors were stimulated with $10^{7}$ killed conidia per $\mathrm{ml}$ of the clinical isolate A. fumigatus V05-27 (ref. 44). After 24-h incubation in the presence of $10 \%$ human pooled serum at $37^{\circ} \mathrm{C}$ and $5 \% \mathrm{CO}_{2}$, supernatants were collected and IL- $1 \beta$ and IL- 8 were measured by ELISA (R\&D Systems and PeliKine, respectively).

Statistical analysis. The probability of invasive aspergillosis resulting from CLEC1A rs2306894 SNP was analysed using the cumulative incidence method and compared using Gray's test ${ }^{45}$. Cumulative incidences were computed with the cmprsk package for R version 2.10.1 (ref. 46), with censoring of data at the date of last follow-up visit and defining relapse and death as competing events. A period of 24 months after transplant was chosen to include all cases of fungal infection. Mouse survival data were analysed with the log rank test using GraphPad Prism. No data was excluded.

In vitro and ex vivo data were analysed using the GraphPad Prism software. Two-tailed student's $t$-tests or Mann-Whitney $U$ tests were used to determine statistical significance. All experiments were independently repeated at least once, unless otherwise indicated.

Data availability. The data that support the findings of this study are available from the corresponding author upon reasonable request.

25. Thau, N. et al. rodletless mutants of Aspergillus fumigatus. Infect. Immun. 62 , 4380-4388 (1994)

26. Tsai, H. F., Wheeler, M. H., Chang, Y. C. \& Kwon-Chung, K. J. A developmentally regulated gene cluster involved in conidial pigment biosynthesis in Aspergillus fumigatus. J. Bacteriol. 181, 6469-6477 (1999).

27. Sarfati, J. et al. A new experimental murine aspergillosis model to identify strains of Aspergillus fumigatus with reduced virulence. Nippon Ishinkin Gakkai Zasshi 43, 203-213 (2002)

28. Graham, L. M. et al. Soluble Dectin-1 as a tool to detect $\beta$-glucans. J. Immunol. Methods 314, 164-169 (2006).

29. Ettinger, R., Browning, J. L., Michie, S. A., van Ewijk, W. \& McDevitt, H. O. Disrupted splenic architecture, but normal lymph node development in mice expressing a soluble lymphotoxin- $\beta$ receptor-lgG1 fusion protein. Proc. Natl Acad. Sci. USA 93, 13102-13107 (1996)

30. Bayry, J. et al. Surface structure characterization of Aspergillus fumigatus conidia mutated in the melanin synthesis pathway and their human cellular immune response. Infect. Immun. 82, 3141-3153 (2014).

31. Pyż, E. et al. Characterisation of murine MICL (CLEC12A) and evidence for an endogenous ligand. Eur. J. Immunol. 38, 1157-1163 (2008)

32. Galfrè, G., Milstein, C. \& Wright, B. Rat $\times$ rat hybrid myelomas and a monoclonal anti-Fd portion of mouse IgG. Nature 277, 131-133 (1979).

33. Willment, J. A., Gordon, S. \& Brown, G. D. Characterization of the human $\beta$-glucan receptor and its alternatively spliced isoforms. J. Biol. Chem. 276, 43818-43823 (2001).

34. Richie, D. L. et al. A role for the unfolded protein response (UPR) in virulence and antifungal susceptibility in Aspergillus fumigatus. PLoS Pathog. 5, e1000258 (2009).

35. Taylor, P. R., Brown, G. D., Geldhof, A. B., Martinez-Pomares, L. \& Gordon, S. Pattern recognition receptors and differentiation antigens define murine myeloid cell heterogeneity ex vivo. Eur. J. Immunol. 33, 2090-2097 (2003).

36. Kerscher, B. et al. Mycobacterial receptor, Clec4d (CLECSF8, MCL), is coregulated with Mincle and upregulated on mouse myeloid cells following microbial challenge. Eur. J. Immunol. 46, 381-389 (2016).

37. Shepardson, K. M. et al. Myeloid derived hypoxia inducible factor 1-alpha is required for protection against pulmonary Aspergillus fumigatus infection. PLOS Pathog. 10, e1004378 (2014).

38. Redelinghuys, P. et al. MICL controls inflammation in rheumatoid arthritis. Ann. Rheum. Dis. 75, 1386-1391 (2016).

39. Liu, Y. et al. Neoglycolipid-based oligosaccharide microarray system: preparation of NGLs and their noncovalent immobilization on nitrocellulose-coated glass slides for microarray analyses. Methods Mol. Biol. 808, 117-136 (2012).

40. Stoll, M. S. \& Feizi, T. Software tools for storing, processing and displaying carbohydrate microarray data. In Proc. Beilstein Symposium on GlycoBioinformatics (ed. Kettner, C.) 123-140 (Beilstein, 2009).

41. De Pauw, B. et al. Revised definitions of invasive fungal disease from the European Organization for Research and Treatment of Cancer/Invasive Fungal Infections Cooperative Group and the National Institute of Allergy and Infectious Diseases Mycoses Study Group (EORTC/MSG) Consensus Group. Clin. Infect. Dis. 46, 1813-1821 (2008). 


\section{LETTER RESEARCH}

42. Kumar, V. et al. Immunochip SNP array identifies novel genetic variants conferring susceptibility to candidaemia. Nat Commun 5, 4675 (2014).

43. Johnson, A. D. et al. SNAP: a web-based tool for identification and annotation of proxy SNPs using HapMap. Bioinformatics 24, 2938-2939 (2008).

44. Netea, M. G. et al. Aspergillus fumigatus evades immune recognition during germination through loss of toll-like receptor-4-mediated signal transduction. J. Infect. Dis. 188, 320-326 (2003).
45. Gray, R. T. A class of $K$-sample tests for comparing the cumulative incidence of a competing risk. Ann. Stat. 16, 1141-1154 (1988)

46. Scrucca, L., Santucci, A. \& Aversa, F. Competing risk analysis using R: an easy guide for clinicians. Bone Marrow Transplant. 40, 381-387 (2007).

47. LeibundGut-Landmann, S., Osorio, F., Brown, G. D. \& Reis e Sousa, C.

Stimulation of dendritic cells via the dectin-1/Syk pathway allows priming of cytotoxic T-cell responses. Blood 112, 4971-4980 (2008). 
a

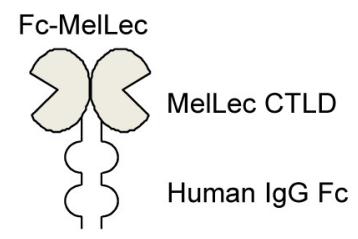

b

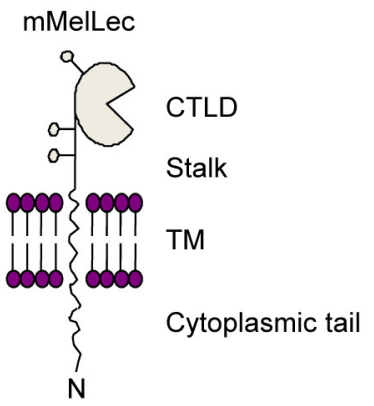

C

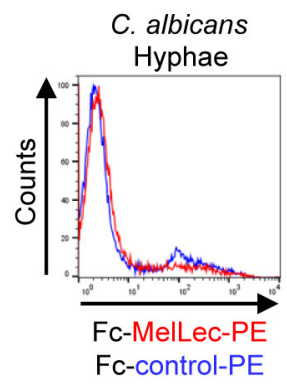

e Cladosporium cladosporioides
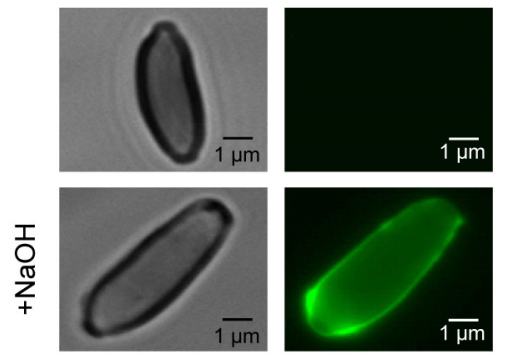

Extended Data Figure $1 \mid$ MelLec recognizes ligands on selected fungi and fungal morphotypes. $\mathbf{a}, \mathbf{b}$, Cartoon representations of the structures of Fc-MelLec (a) and the full-length receptor (b). Lollipop structures represent predicted glycosylation sites. c, Fc-MelLec or FcCLEC12B (Fc-control) staining of C. albicans hyphae, generated in RPMI with $10 \%$ fetal bovine serum for $90 \mathrm{~min}$. Fungal particles were analysed by flow cytometry. Experiment was repeated independently twice, with similar results. d, Representative light microscopy images and immunofluorescence micrographs using Fc-MelLec or antigalactomannan (GM, control) as probes to detect ligands on A. fumigatus mycelium. Experiments were repeated three times independently,

\section{d fumigatus}

Mycelium

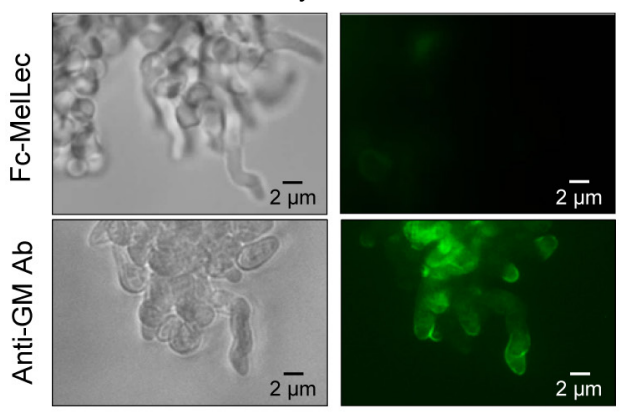

f

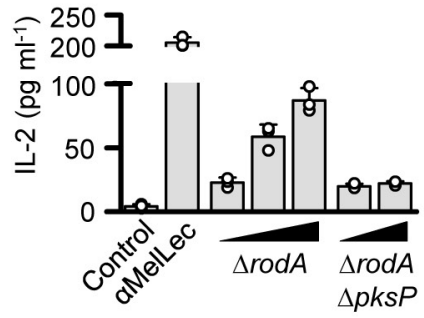

with similar results. e, Representative light microscopy images and immunofluorescence micrographs showing the surface distribution of MelLec ligands on C. cladosporioides using Fc-MelLec as a probe. The lower panels show fungal cells after treatment with $1 \mathrm{M} \mathrm{NaOH}$. Experiments were repeated three times independently, with similar results. f, IL-2 production by MelLec-expressing BWZ reporter cells after stimulation by anti-MelLec antibody ( $\alpha$ MelLec) crosslinking or with $\Delta \operatorname{rod} A(1: 1,5: 1,10: 1)$ or $\Delta \operatorname{rod} A \Delta p k s P(5: 1,10: 1)$ A. fumigatus conidia, as indicated. Values shown are mean \pm s.d. Experiment was repeated three times independently, with similar results. 


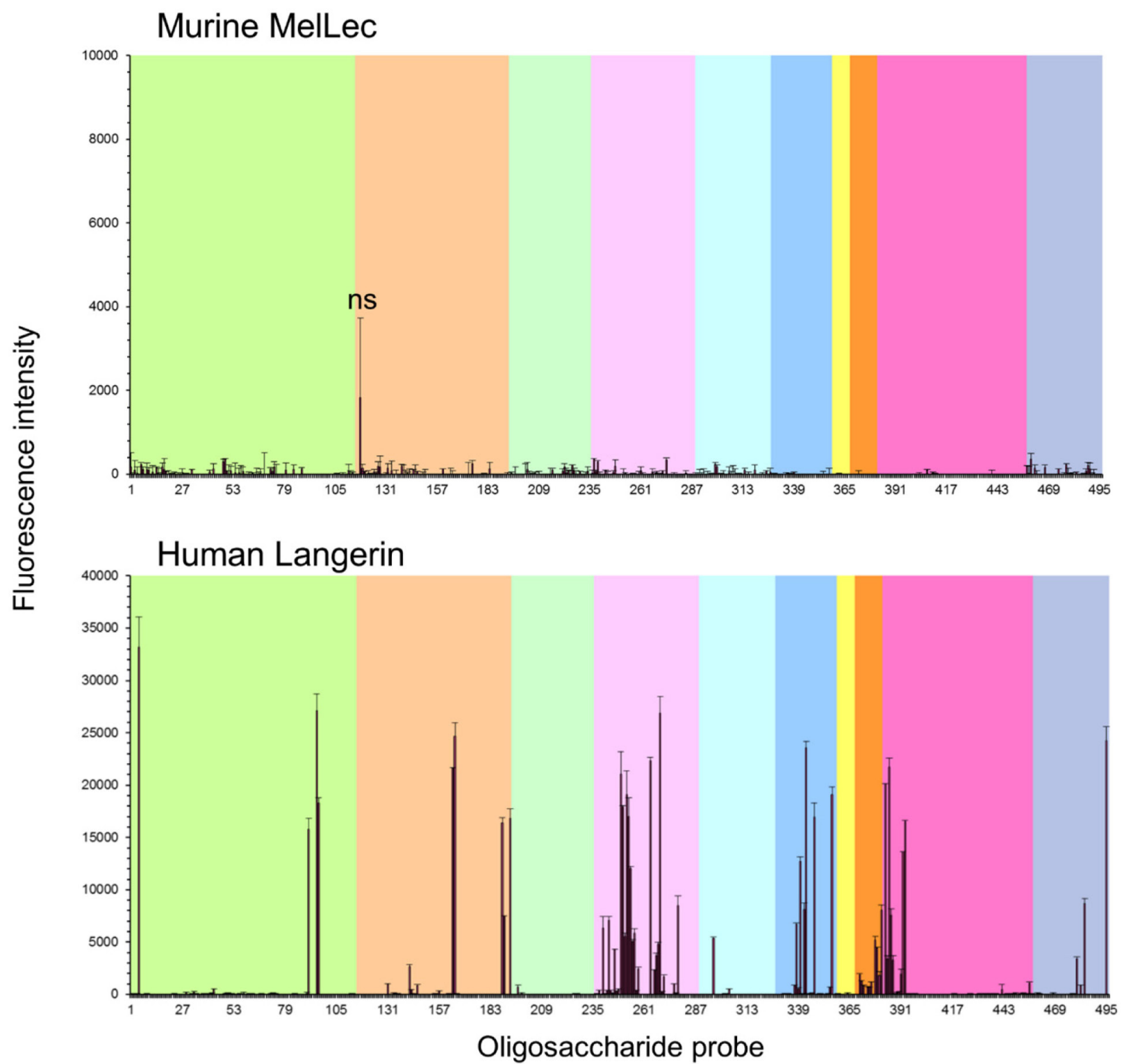

$\square$ Lac \& LacNAC-based $\square$ LNnT \& LNT-based $\square$ PolyLac li-active $\square$ N-glycans

$\square$ Ganglioside $\square$ o-glycans $\square$ Poly-sialyı $\square$ gags $\square$ Homo-oligomers $\square$ Misc.

Extended Data Figure 2 | Glycan microarray analyses of mouse Fc-MelLec and human langerin. These 496 lipid-linked probes are arranged according to their backbone sequences as annotated in the coloured panels below the figure. GAGs, glycosaminoglycans; Lac, lactose; LacNAc, $\mathrm{N}$-acetyllactosamine; LNnT, lacto- $\mathrm{N}$-neotetraose; LNT, lacto- $\mathrm{N}$-tetraose;
Misc., miscellaneous; PolyLac, polylactosamine. The signals are means of fluorescence intensities of duplicate spots, printed at $5 \mathrm{fM}$ per spot level with error bars representing half of the difference between the two values. The signals shown together with the probe sequences are in Supplementary Table 1. NS, not significant. 
a
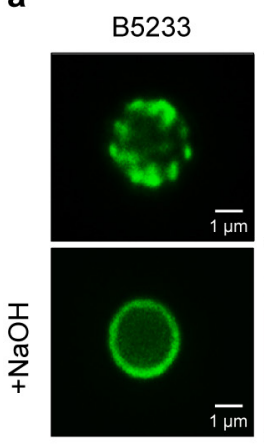

CBS386.75

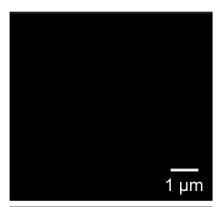

CBS110.46

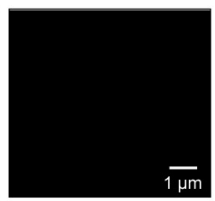

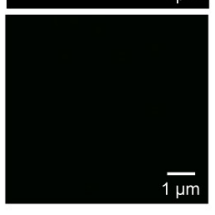

$\Delta p k s P$
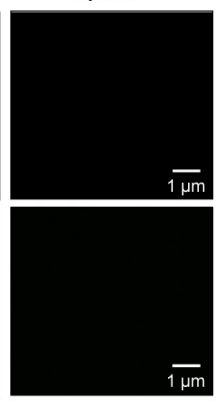

b

$\Delta \operatorname{rod} A \Delta k s \mathrm{P}$

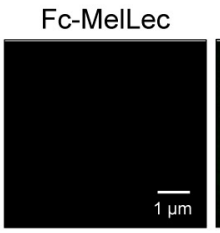

ConA-FITC
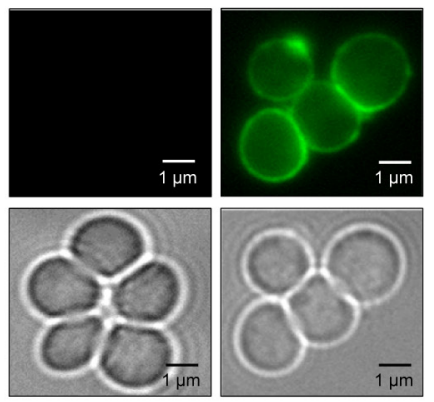

C

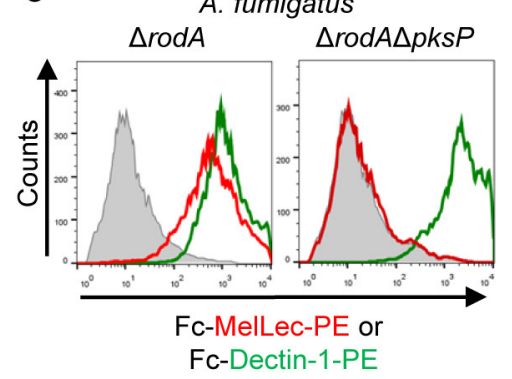

d

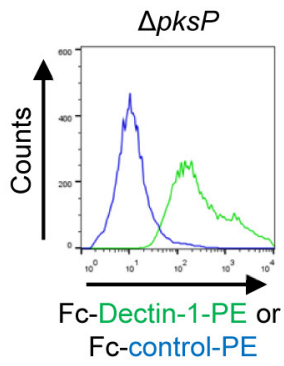

e

\section{A. fumigatus}

Melanin ghosts

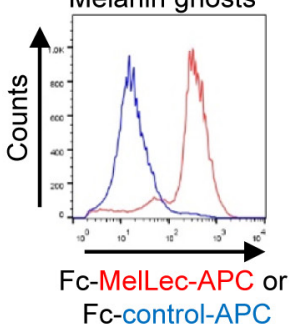

f

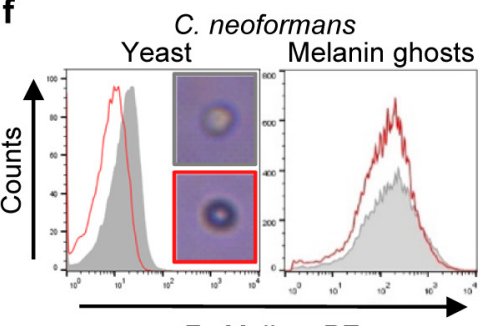

Fc-MelLec-PE

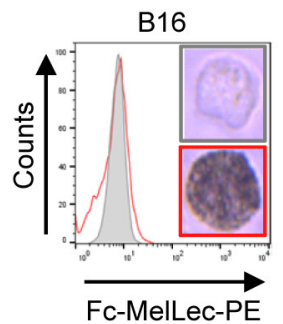

Extended Data Figure $3 \mid$ MelLec recognizes DHN-melanin.

a, Representative immunofluorescence micrographs using Fc-MelLec as a probe showing the surface distribution of MelLec-ligands on wild-type A. fumigatus and various melanin-deficient mutants. Lower panels show conidia treated with $1 \mathrm{M} \mathrm{NaOH}$. b, Representative immunofluorescence micrographs and light microscopy images of $\Delta \operatorname{rod} A \Delta p k s P$ A. fumigatus conidia stained with Fc-MelLec or ConA-FITC. c, Representative histograms showing the presence or absence of MelLec or Dectin-1 ligands on $\triangle \operatorname{rod} A$ or $\Delta \operatorname{rod} A \Delta p k s P A$. fumigatus conidia. Fungal particles were stained with Fc-MelLec (red) or Fc-Dectin-1 (green) and analysed by flow cytometry. Grey histograms indicate secondary-only control. d, Representative histogram showing the presence of Dectin-1 ligands on $\triangle p k s P$ A. fumigatus conidia. Fungal particles were stained with Fc-Dectin-1 (green) or Fc-CLEC12B (Fc-control; blue) and analysed by flow cytometry. e, Representative histogram showing the presence of MelLec ligands on melanin ghosts of $A$. fumigatus conidia. Fungal particles were stained with Fc-MelLec (red) or Fc-CLEC12B (Fc-control; blue) and analysed by flow cytometry. In a-e, experiments were repeated three times independently, with similar results. f, Flow-cytometric analysis of melanized (red) and non-melanized (grey) Cryptococcus neoformans yeast and melanin ghosts, and B16 melanoma cells ${ }^{47}$, stained with Fc-MelLec. The experiment was repeated independently twice, with similar results. 
a

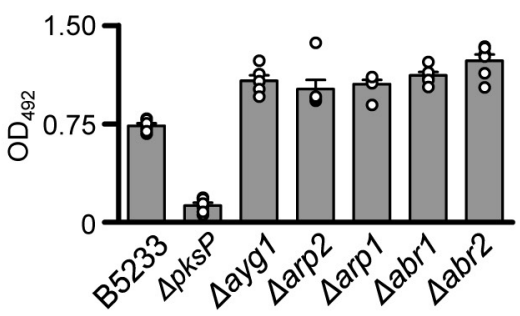

C $85233 \mathrm{NaOH}$-treated

Fc-MelLec control
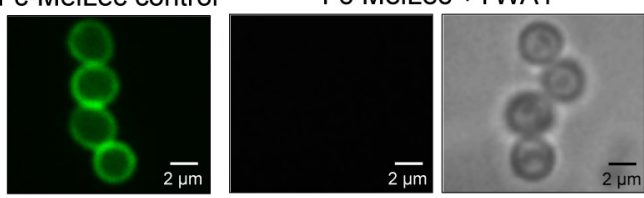

e

1,8-DHN

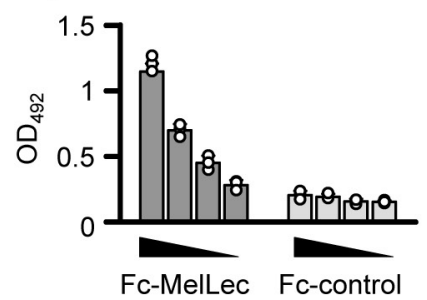

b

f

1,4-DHN
B5233 NaOH-treated

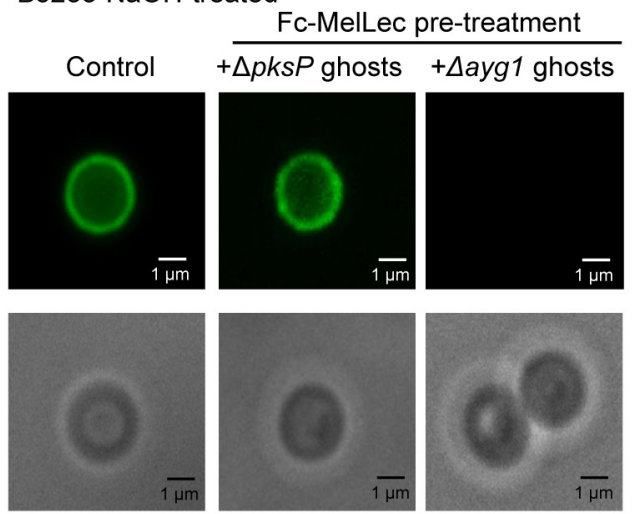

d

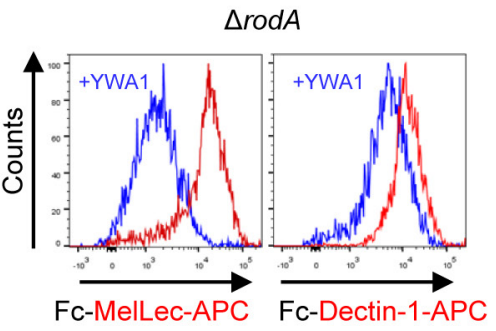

$1,2-\mathrm{DHN}$

g

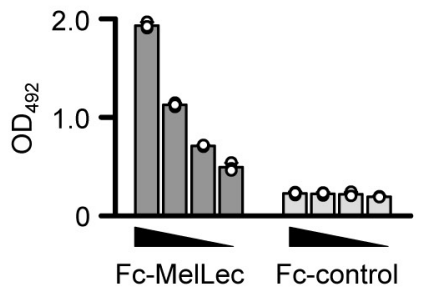

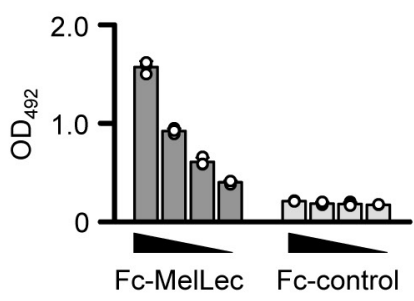

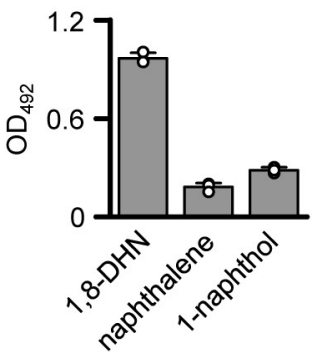

Extended Data Figure $4 \mid$ MelLec recognizes naphthalene-diol.

a, Detection of MelLec ligands in ghosts of melanin mutants of

A. fumigatus by ELISA, as indicated. Values show mean \pm s.d.

b, Representative immunofluorescence and light micrograph images of Fc-MelLec ligands on $\mathrm{NaOH}$-treated A. fumigatus B5233 conidia after pretreatment with ghosts of $\Delta p k s P$ or $\triangle a y g 1$ conidia, as indicated. c, Representative immunofluorescence micrographs and light microscopy images of MelLec ligands on $\mathrm{NaOH}$-treated wild-type A. fumigatus conidia after pretreatment with or without YWA1. In a-c, experiments were repeated at least three times independently, with similar results. d, Detection of Fc-MelLec or Fc-Dectin-1 ligands on $\triangle \operatorname{rodA}$ conidia after pretreatment with (blue) or without (red) YWA1. The experiment was repeated independently twice, with similar results. e, f, Detection of 1,8-DHN (e) and 1,2-DHN and 1,4-DHN (f) by Fc-MelLec and $\mathrm{Fc}$-control using ELISA. Values show mean \pm s.d. g, Detection of 1,8-DHN, naphthalene and 1-naphthol by Fc-MelLec using ELISA. Values show mean \pm s.d. In e-g, experiments were repeated at least three times independently, with similar results. 
a

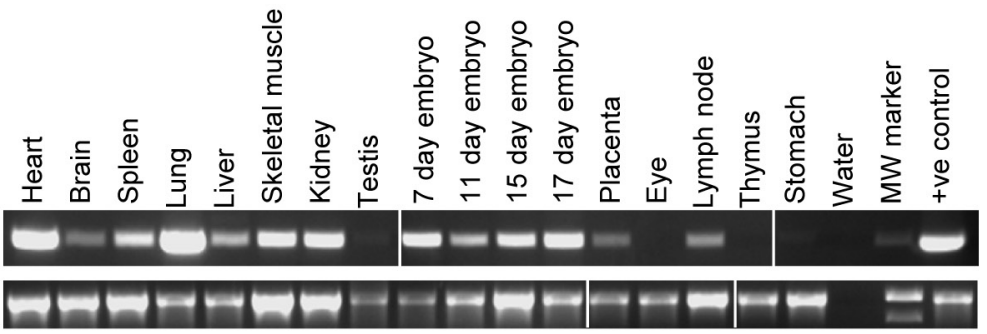

b
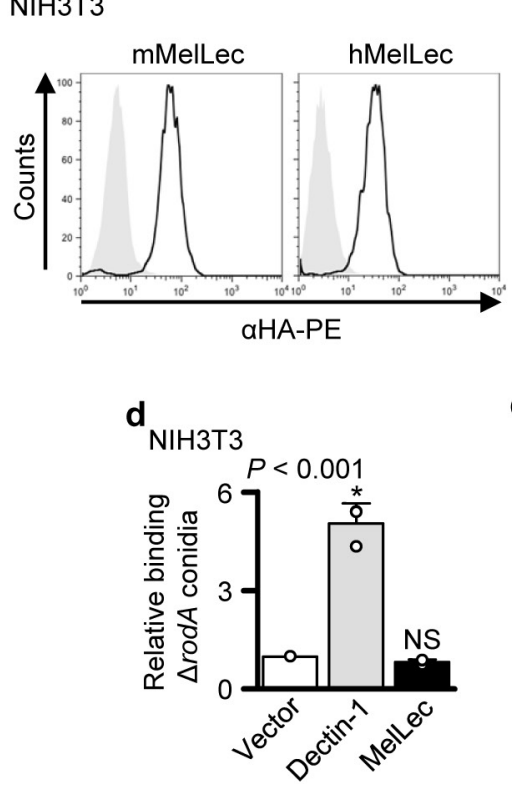

Extended Data Figure $5 \mid$ MelLec is expressed at the cell surface. a, RT-PCR detection of MelLec expression in various tissues. The expression of glyceraldehyde-3-phosphate dehydrogenase (G3PDH) in these samples, also used for the characterization of $\mathrm{MCL}^{36}$, is shown as a control. The experiment was performed once; for gel source data, see Supplementary Fig. 1. b, Flow-cytometric analysis of surface expression of haemagglutinin-tagged mouse (m) and human (h) MelLec on the surface of NIH3T3 fibroblasts (black open histograms). NIH3T3 cells transfected with vector only served as controls (grey filled histograms). c, Westernblot analysis of lysates of haemagglutinin-tagged MelLec expressing

e
$\mathrm{NIH} 3 \mathrm{T3}$
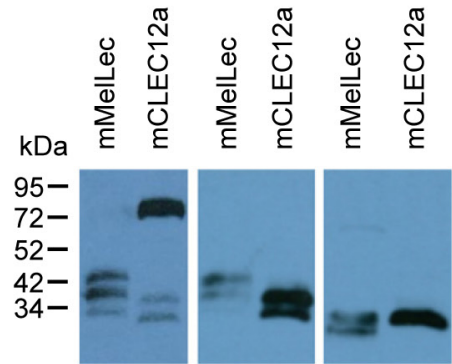

$\mathrm{N}$-glycosidase

$\beta$-mercaptoethanol NIH3T3

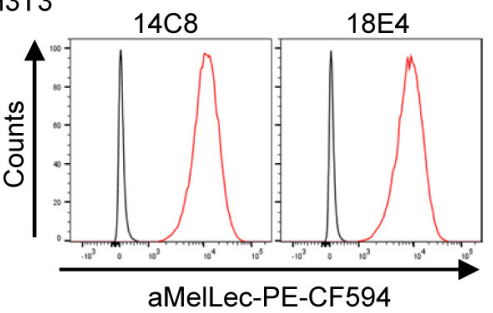

NIH3T3 cells under reducing and non-reducing conditions and with $(+)$ and without $(-) \mathrm{N}$-glycosidase. Haemagglutinin-tagged CLEC12A (ref. 31) expressing NIH3T3 cells served as controls (for blot source data, see Supplementary Fig. 1). d, Relative binding of FITC-labelled $\Delta$ rodA A. fumigatus conidia to NIH3T3 cells transduced with vector only, Dectin-1 or MelLec, as determined by flow cytometry. Values shown are mean \pm s.d., analysed by one-way ANOVA. e, Screening of hybridoma supernatants on MelLec-expressing (red) and parental (black) NIH3T3 cells. In $\mathbf{b}-\mathbf{e}$, experiments were repeated at least three times independently, with similar results. $* P \leq 0.05$; NS, not significant. 
a

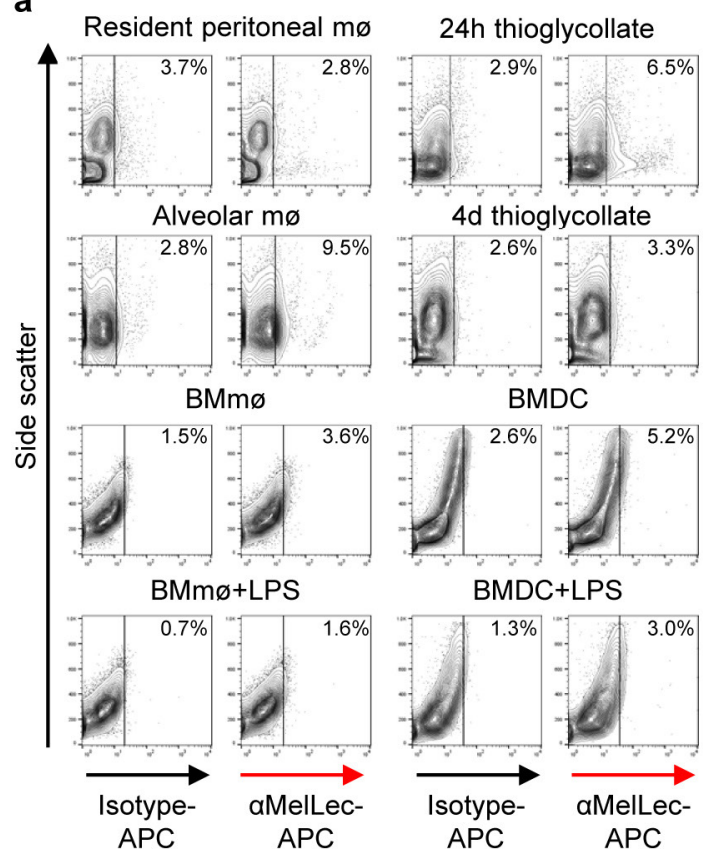

b

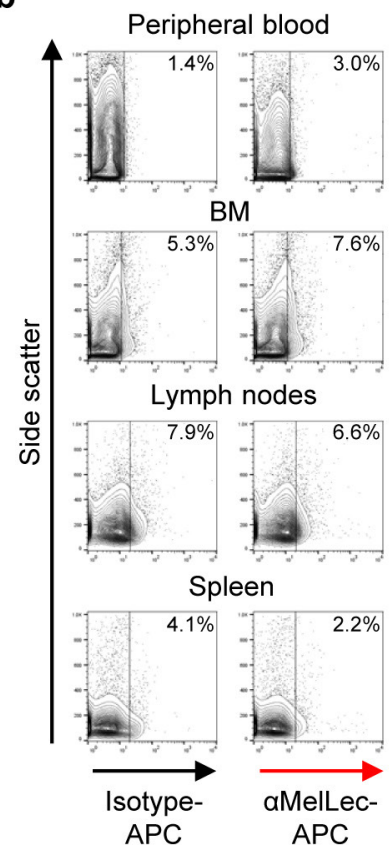

C

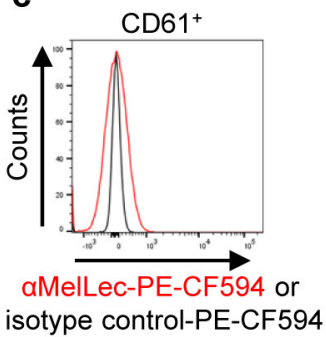

Extended Data Figure 6 | Mouse MelLec is not expressed by myeloid cells. a, b, Flow-cytometric analysis of MelLec expression on various ex vivo and in vitro derived myeloid cells (a) and peripheral blood, bone marrow, lymph nodes and spleen (b). Experiments were repeated at least

twice independently, with similar results. c, Flow-cytometric analysis of MelLec expression on $\mathrm{CD}^{+} 1^{+}$platelets. Experiment was repeated at least three times independently, with similar results. BM, bone marrow; DC, dendritic cell; LPS, lipopolysaccharide; mø, macrophage. 


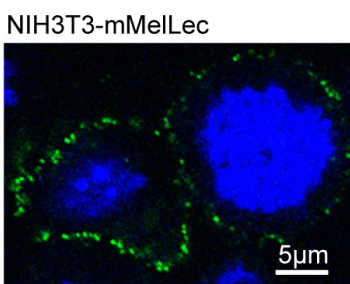

NIH3T3-control

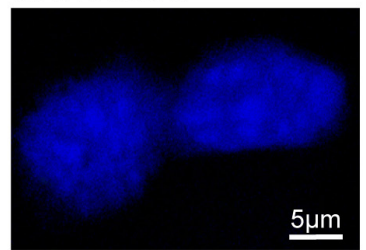

b

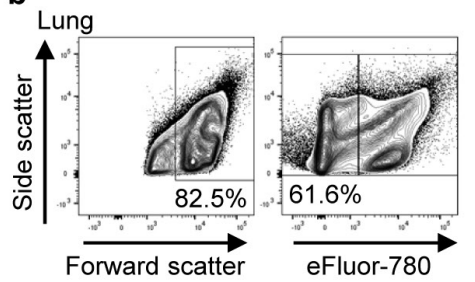

C $_{\text {Liver, CD45- }}$
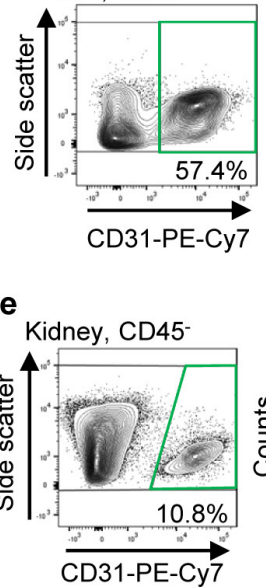

h

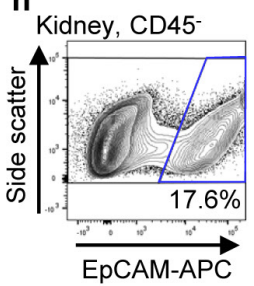

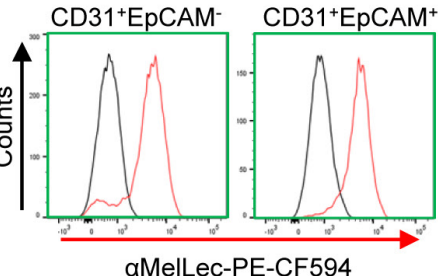

aMelLec-PE-CF594 d

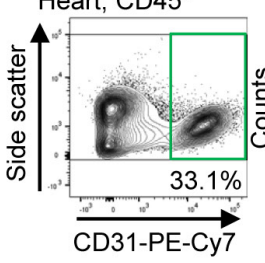

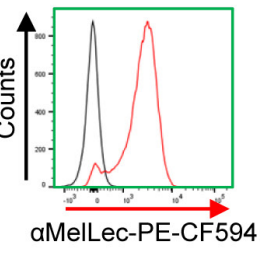

f

Small intestine, CD45-
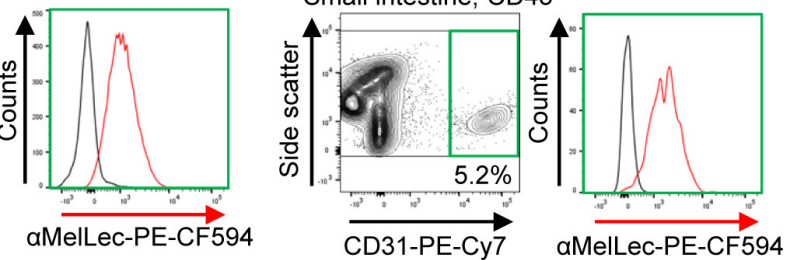

g

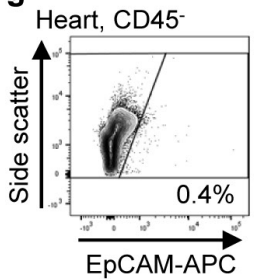

i

Small intestine, CD45-
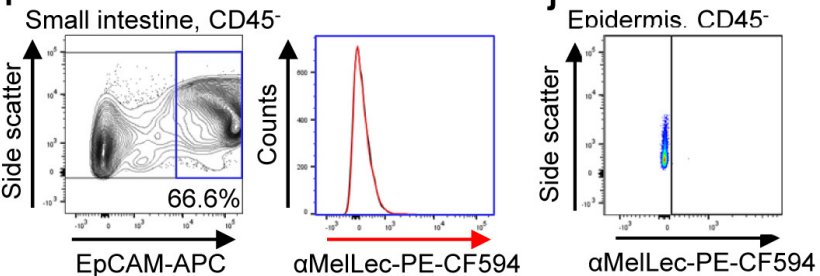

$\mathbf{k}_{\text {Mouse Clec1a genomic locus }}$

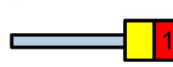

Targeting vector

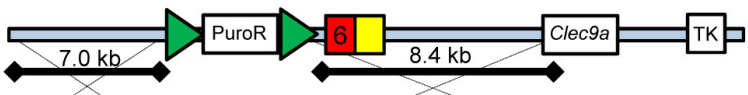

Targeted allele

Knockout allele
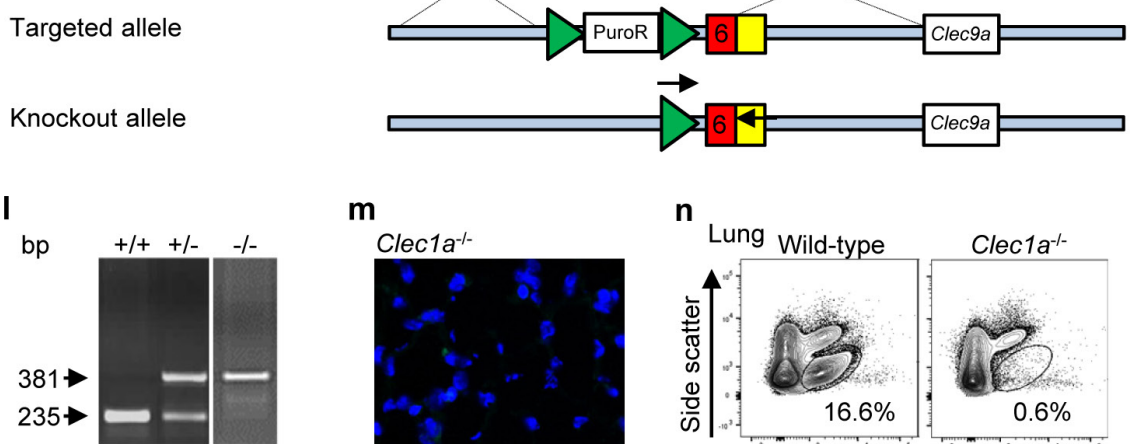

m

Clec1 $a^{-1-}$

n
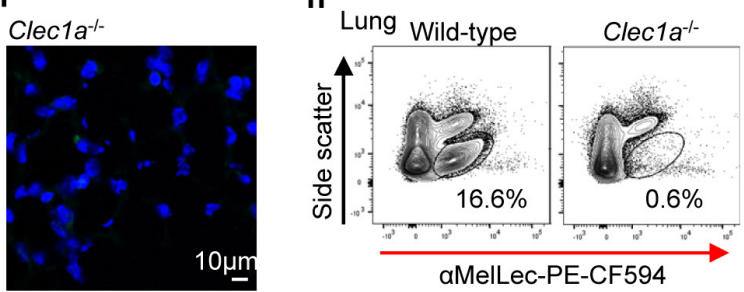

Extended Data Figure 7 See next page for caption. 


\section{LETTER RESEARCH}

Extended Data Figure $7 \mid$ MelLec expression in tissues and generation of $\mathrm{Clec} \mathrm{Ia}^{-1-}$ mice. a, Immunofluorescence microscopy of MelLecexpressing versus control NIH3T3 cells labelled with anti-MelLec antibody (green). Nuclei are stained with DAPI (blue). Experiments were repeated at least three times independently, with similar results. b, Exemplar flowcytometric gating strategy for identification of live cells from tissue. c, Flow-cytometric analysis of MelLec expression on live CD $45^{-} \mathrm{CD} 31^{+}$ $\mathrm{EpCAM}^{-}$and $\mathrm{EpCAM}^{+}$populations in the liver. $\mathbf{d}-\mathbf{j}$, Flow cytometric analysis of MelLec expression on live $\mathrm{CD}_{4} 5^{-} \mathrm{CD} 31^{+}$cells in the heart (d), kidney (e) and small intestine (f), and on live CD45-EpCAM ${ }^{+}$cells in the heart (g), kidney (h), small intestine (i) and epidermis (j). In $\mathbf{b}-\mathbf{j}$, experiments were repeated at least twice independently, with similar results. Black lines, isotype controls. $\mathbf{k}$, Schematic of the wild-type Clec1a locus, gene targeting vector, PCR primer sites and correctly targeted recombinant allele. 1, PCR analysis of gene-targeted mice (for gel source data, see Supplementary Fig. 1). +/+, wild-type, +/- heterozygous and $-1-$ homozygous for the targeted allele. $\mathbf{m}$, Immunofluorescence microscopy of naive lung tissue from $\mathrm{Clecla}^{-1-}$ mice (labelling of wild-type lung is shown in Fig. 3b). n, Analysis of MelLec expression in disaggregated lung tissue from wild-type (wt) or Clec1 $a^{-/-}$mice by flow cytometry. In $\mathbf{l}-\mathbf{n}$, experiments were repeated at least three times independently, with similar results. 
a
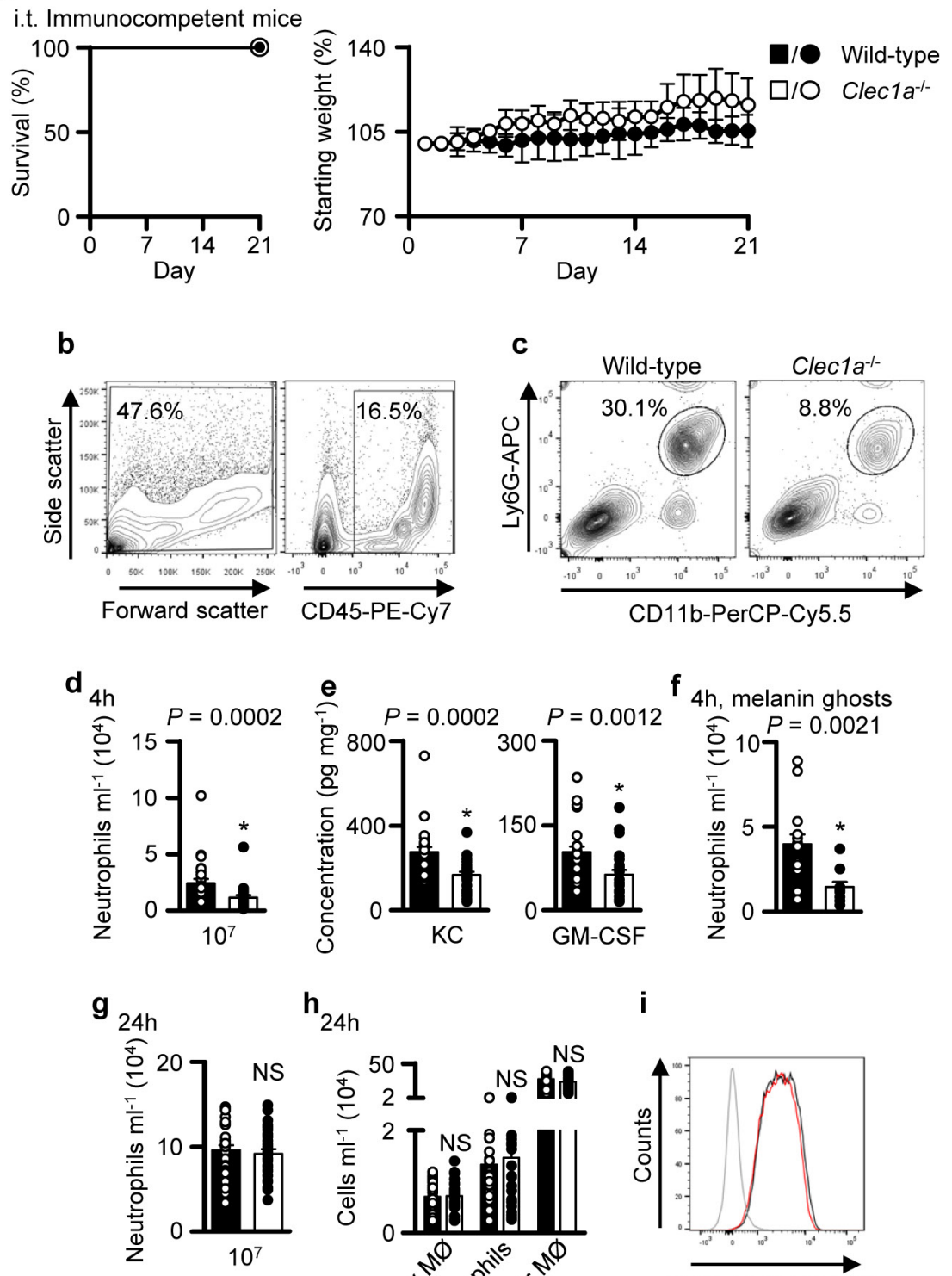

$\mathrm{h}_{24 \mathrm{~h}}$

i
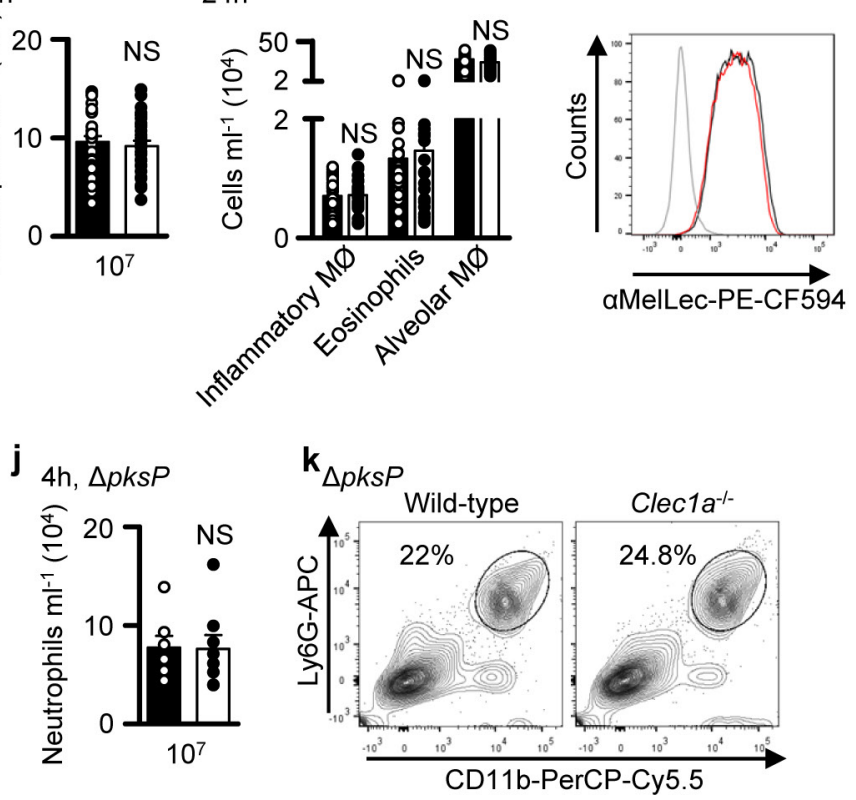

Extended Data Figure 8 | See next page for caption. 
Extended Data Figure 8| Clec1a $^{-1-}$ mice show early inflammatory defects upon challenge with $A$. fumigatus. a, Survival (left) and weight measurements (right) of immunocompetent mice after i.t. infection with $10^{7}$ A. fumigatus conidia ( $n=4$ mice per group). Values shown are mean \pm s.d. b, Exemplar flow-cytometric gating strategy for the identification of $\mathrm{CD} 45^{+}$cells from bronchoalveolar lavage.

c, Representative FACS profiles of pulmonary CD $11 b^{+}$Ly $6 \mathrm{G}^{\text {high }}$ neutrophils in wild-type and $\mathrm{Clec} 1 \mathrm{a}^{-1-}$ mice $4 \mathrm{~h}$ after challenge with $10^{7}$ A. fumigatus conidia (wild-type $n=29$ mice; Clec1 $a^{-1-} n=26$ mice). d, e, Pulmonary CD $11 \mathrm{~b}^{+}$Ly6 $6 \mathrm{G}^{\text {high }}$ neutrophils (wild-type $n=29$ mice, Clec $1 a^{-1-} n=26$ mice) (d) and cytokines ( $n=25$ mice per group) (e) in mice $4 \mathrm{~h}$ after challenge with $10^{7} \mathrm{~A}$. fumigatus conidia. f, Pulmonary $\mathrm{CD}_{11 \mathrm{~b}}{ }^{+}$Ly6G $\mathrm{G}^{\text {high }}$ neutrophils in mice $4 \mathrm{~h}$ after challenge with melanin

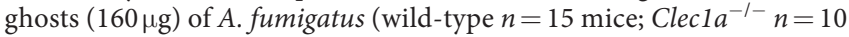
mice). Samples with blood contamination were excluded. g, Pulmonary $\mathrm{CD}_{11 \mathrm{~b}^{+}}{\text {Ly } 6 \mathrm{G}^{\text {high }}}$ neutrophils in mice $24 \mathrm{~h}$ after challenge with

$10^{7}$ A. fumigatus conidia (wild-type $n=33$ mice, Clec $1 a^{-1-} n=30$ mice). h, Cellular inflammatory profiles of mice $24 \mathrm{~h}$ after challenge with $10^{7}$ A. fumigatus conidia (wild-type $n=33$ mice, Clec1 $a^{-1-} n=30$ mice). Alveolar macrophages were defined as $\mathrm{CD} 11 \mathrm{c}^{+}$Siglec- $\mathrm{F}^{+}$, inflammatory macrophages as CD $11 b^{+} \mathrm{F} 4 / 80^{+}$and eosinophils as $\mathrm{CD} 11 \mathrm{~b}^{+}$Siglec- $\mathrm{F}^{+}$. In $\mathbf{d}-\mathbf{h}$, values shown are mean \pm s.e.m. of pooled data from at least two independent experiments, analysed by two-sided Mann-Whitney $U$ test. i, Expression of MelLec on pulmonary $\mathrm{CD} 45^{-} \mathrm{CD} 31^{+}$cells isolated from uninfected mice (black) and mice $24 \mathrm{~h}$ after infection with A. fumigatus conidia (red). Grey line, isotype control $(n=3$ mice per group). j, Pulmonary CD $11 \mathrm{~b}^{+}$Ly $6 \mathrm{G}^{\text {high }}$ neutrophils in mice $4 \mathrm{~h}$ after challenge with $10^{7} \Delta p k s P$ A. fumigatus conidia (wild-type $n=7$ mice, Clec $1 a^{-1-} n=8$ mice). Values shown are mean \pm s.e.m. of pooled data from two independent experiments, analysed by two-sided MannWhitney $U$ test. $\mathbf{k}$, Representative FACS profiles of pulmonary CD $11 \mathrm{~b}^{+}$ Ly $6 \mathrm{G}^{\text {high }}$ neutrophils in wild-type and Clec $1 a^{-1-}$ mice $4 \mathrm{~h}$ after challenge with $10^{7} \Delta p k s P$ A. fumigatus conidia (wild-type $n=7$ mice, $\mathrm{Clecla}^{-1-}$ $n=8$ mice). $* P \leq 0.05$; NS, not significant. 
a

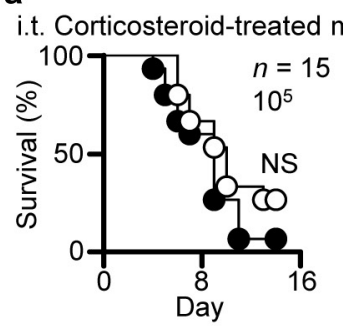

b

Day 4
C

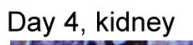

\section{d}

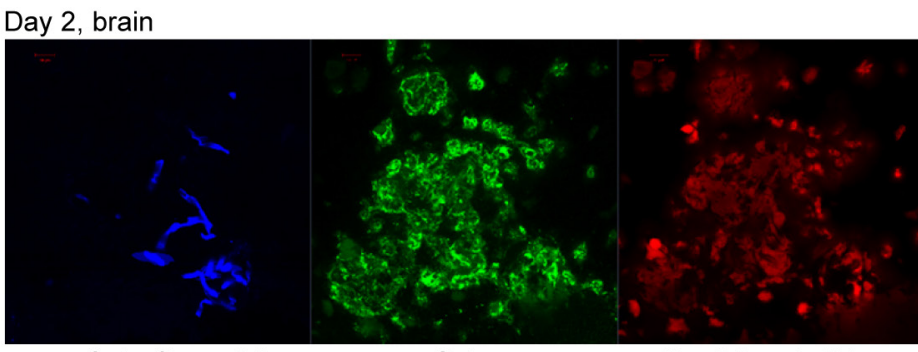

Calcofluor white

Gr1

Propidium iodide

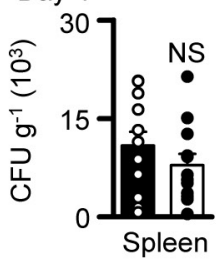

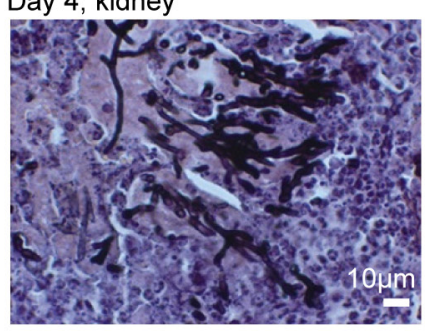

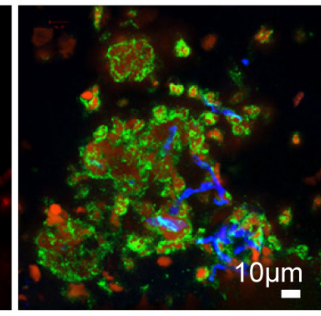

Merge

e Day 4, $\triangle p k s P$

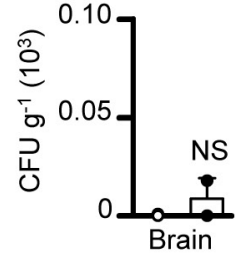

]$_{0.5-1.0}^{1.0}$

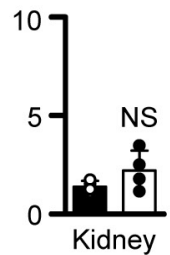

Wild-type

Clec1 $a^{-1-}$
Extended Data Figure 9 $\mid$ Clec1 $^{-1-}$ mice show alterations in antifungal immunity during systemic infection. a, Survival of corticosteroid-treated mice after i.t. infection with $10^{5} \mathrm{~A}$. fumigatus conidia $(n=15$ mice per group). Pooled data from two independent experiments, analysed by logrank test. b. Fungal burdens in various mouse tissues, as indicated, 4 days after i.v. infection with $10^{6} \mathrm{~A}$. fumigatus conidia ( $n=12$ mice per group). Values shown are mean \pm s.e.m. of pooled data from two independent experiments, analysed by two-sided Mann-Whitney $U$ test. c, Tissue section of kidney from day-4-infected $\mathrm{Clecla}^{-/-}$mouse stained with
Grocott's methenamine silver stain and haematoxylin ( $n=3$ mice per group). d, Immunofluorescence microscopy of brain from day-2-infected Clec1 $a^{-1-}$ mouse ( $n=3$ mice per group). Fungi are stained with calcofluor white (blue), leukocytes with Gr1 (green) and DNA with propidium iodide (red). e, Fungal burdens in various mouse tissues, as indicated, four days after i.v. infection with $10^{6} \Delta p k s P$ A. fumigatus conidia $(n=4$ mice per group). Values shown are mean \pm s.d., analysed by two-sided MannWhitney $U$ test. ${ }^{*} P \leq 0.05$; NS, not significant. 
a

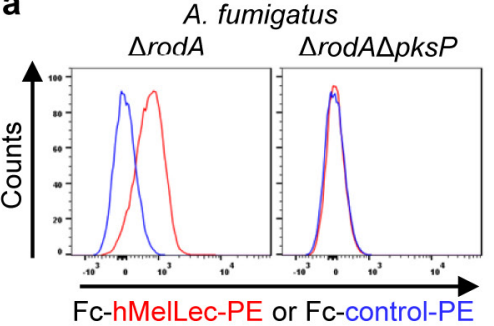

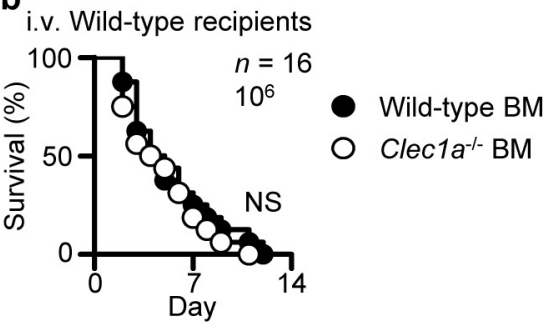

d

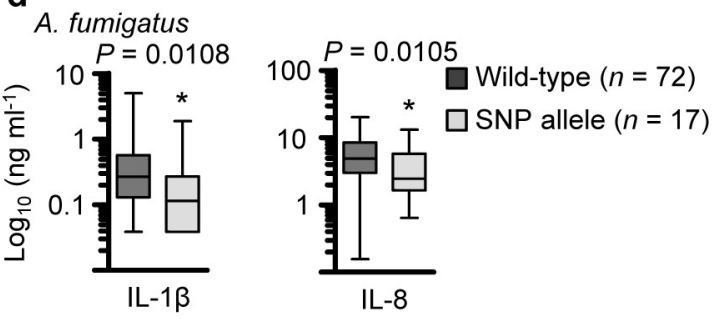

e RAW264.7 macrophages
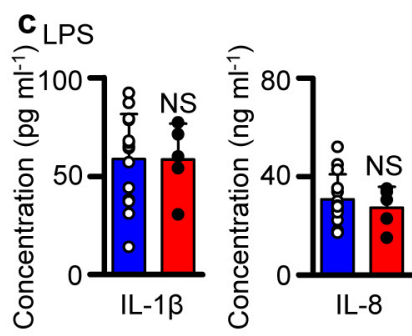

Wild-type $(n=14)$

SNP allele $(n=5)$

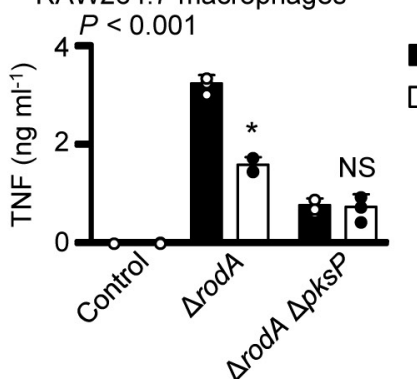

Wild-type

SNP (G26A)

Extended Data Figure 10 | A single nucleotide polymorphism in human MelLec influences anti-Aspergillus inflammatory responses. a, Representative histograms showing the presence or absence of human MelLec ligands on $\triangle \operatorname{rod} A$ or $\Delta \operatorname{rod} A \Delta p k s P$ A. fumigatus conidia, as determined by flow cytometry. Fc-CLEC12B was used as a control (Fc-control). The experiment was repeated at least three times independently, with similar results. b, Survival of irradiated wild-type mice reconstituted with wild-type or Clec1 $a^{-1-}$ bone marrow (BM), as indicated, after i.v. infection with $10^{6}$ A. fumigatus conidia ( $n=16$ mice per group). Pooled data from two independent experiments, analysed by log-rank test. c, Inflammatory cytokine production in monocyte-derived macrophages isolated from genotyped individuals, after stimulation with lipopolysaccharide (wild-type $n=14$ individuals,
SNP allele $n=5$ individuals). Values shown are mean \pm s.d., analysed by two-sided Mann-Whitney $U$ test. d, Inflammatory cytokine production in peripheral blood mononuclear cells isolated from genotyped Dutch individuals, after stimulation with heat-killed A. fumigatus conidia (wild-type $n=72$ individuals, SNP allele $n=17$ individuals). Boxes represent the median values and interquartile ranges; whiskers represent minimum and maximum values, analysed by two-sided Mann-Whitney $U$ test. e, Inflammatory cytokine production in transduced RAW264.7 macrophages expressing wild-type or SNP allele after stimulation with $\triangle \operatorname{rodA}$ or $\triangle \operatorname{rod} A \triangle p k s P A$. fumigatus conidia, as indicated. Values shown are mean \pm s.d., analysed by one-way ANOVA and repeated at least three times independently, with similar results. $* P \leq 0.05$; NS, not significant. 


\section{Life Sciences Reporting Summary}

Nature Research wishes to improve the reproducibility of the work that we publish. This form is intended for publication with all accepted life science papers and provides structure for consistency and transparency in reporting. Every life science submission will use this form; some list items might not apply to an individual manuscript, but all fields must be completed for clarity.

For further information on the points included in this form, see Reporting Life Sciences Research. For further information on Nature Research policies, including our data availability policy, see Authors \& Referees and the Editorial Policy Checklist.

\section{- Experimental design}

\section{Sample size}

Describe how sample size was determined.

\section{Data exclusions}

Describe any data exclusions.

\section{Replication}

Describe whether the experimental findings were reliably reproduced.

4. Randomization

Describe how samples/organisms/participants were allocated into experimental groups.

5. Blinding

Describe whether the investigators were blinded to group allocation during data collection and/or analysis.
Sample sizes of at least five per group were chosen as this would allow the detection of a $25 \%$ difference in the mean between experimental and control groups with a probability of greater than $95 \%(p<0.05)$, assuming a standard deviation of around $15 \%$ and a minimum power value of 0.8 .

There were no data exclusions except for data shown in Extended figure 10c where samples with blood contamination were excluded. This is indicated in the manuscript.

All experiments were independently replicated at least once.

Mice were randomly assigned to experimental or control groups, co-housed, and experiments were not blinded.

Mice were randomly assigned to experimental or control groups, co-housed, and experiments were not blinded.

Note: all studies involving animals and/or human research participants must disclose whether blinding and randomization were used.

\section{Statistical parameters}

For all figures and tables that use statistical methods, confirm that the following items are present in relevant figure legends (or in the Methods section if additional space is needed).

n/a Confirmed

$\bigotimes$ The exact sample size $(n)$ for each experimental group/condition, given as a discrete number and unit of measurement (animals, litters, cultures, etc.)

A description of how samples were collected, noting whether measurements were taken from distinct samples or whether the same sample was measured repeatedly

$\bigotimes$ A statement indicating how many times each experiment was replicated

The statistical test(s) used and whether they are one- or two-sided (note: only common tests should be described solely by name; more complex techniques should be described in the Methods section)

$\bigotimes$ A description of any assumptions or corrections, such as an adjustment for multiple comparisons

$\bigotimes$ The test results (e.g. $P$ values) given as exact values whenever possible and with confidence intervals noted

$\bigotimes$ A clear description of statistics including central tendency (e.g. median, mean) and variation (e.g. standard deviation, interquartile range) Clearly defined error bars 
Policy information about availability of computer code

\section{Software}

Describe the software used to analyze the data in this study.

For manuscripts utilizing custom algorithms or software that are central to the paper but not yet described in the published literature, software must be made available to editors and reviewers upon request. We strongly encourage code deposition in a community repository (e.g. GitHub). Nature Methods guidance for providing algorithms and software for publication provides further information on this topic.

\section{- Materials and reagents}

Policy information about availability of materials

8. Materials availability

Indicate whether there are restrictions on availability of unique materials or if these materials are only available for distribution by a for-profit company.

9. Antibodies

Describe the antibodies used and how they were validated for use in the system under study (i.e. assay and species).

10. Eukaryotic cell lines

a. State the source of each eukaryotic cell line used.

b. Describe the method of cell line authentication used.

c. Report whether the cell lines were tested for mycoplasma contamination.

d. If any of the cell lines used are listed in the database of commonly misidentified cell lines maintained by ICLAC, provide a scientific rationale for their use. no restrictions

Streptavidin-PE-CF594, anti-CD45.2-FITC (Clone 104), biotinylated anti-CD61 (clone 2C9.G2), anti-CD326-APC (EpCAM; Clone G8.8), anti-CD31-PE-Cyanine7 (PECAM-1; Clone 390), Ly6G-APC (clone 1A8), CD11b-PE-Cy7 (clone M1/70), CD11c-PerCPCy5.5 (clone HL3), Siglec-F-BV421 (clone E50-2440) and F4/80-AF700 (clone CL:A3-1) and isotype control AFRC MAC 49 (ECACC 85060404; isotype for antiMelLec). All were purchased commercially from all from BD Biosciences, eBioscience or Abcam.

Anti-MelLec antibodies were generated and validated as follows. Sprague Dawley rats were immunised with Fc-MelLec in Freund's complete adjuvant. After a final intraperitoneal boost, without adjuvant, rat splenocytes were harvested and fused with Y3 myeloma cells, as described. Hybridoma supernatants were screened by ELISA and positives were then tested by immunohistochemistry and flow cytometry, as described below, against Fc-MelLec as well as MelLec transduced NIH3T3 fibroblasts.

293T, NIH3T3, RAW265.7 cell lines were originally purchased from the ATTC

No authentication methods were used.

Mycoplasma tests were done historically on these cell lines, but not for the experiments detailed in the manuscript.

none are listed as misidentified

\section{- Animals and human research participants}

Policy information about studies involving animals; when reporting animal research, follow the ARRIVE guidelines

\section{Description of research animals}

Provide details on animals and/or animal-derived materials used in the study.
C57BL/6 and Clec1a-/- mice (8-12 weeks old) were obtained from the specific pathogen-free facility at the University of Aberdeen. Animal experiments were performed using age-matched female mice and conformed to the animal care and welfare protocols approved by UK Home Office (project license 70/8073) in compliance with all relevant local ethical regulations. Clec1a-/- mice were generated commercially (TaconicArtemis) by conventional gene targeting in C57BL/6 embryonic stem cells as detailed in Extended data Fig. 7. 


\section{Description of human research participants}

Describe the covariate-relevant population characteristics of the human research participants.
A total of 310 hematologic patients undergoing allogeneic hematopoietic stem cell transplantation at the Hospital of Santa Maria, Lisbon and Instituto Português de Oncologia (IPO), Porto, between 2009 and 2014 were enrolled in the study. The cases of invasive aspergillosis were identified and classified as "probable" or "proven" according to the revised standard criteria from the European Organization for Research and Treatment of Cancer/Mycology Study Group (EORTC/MSG)41. Exclusion criteria included diagnosis of "possible" invasive aspergillosis, infection with invasive moulds other than Aspergillus spp. or history of pre-transplant mould infection. Study approval was obtained from the institutional review boards (SECVS-125/2014, HSM-632/14 and CES.26/015) and from the National Data Protection Commission (CNPD, 1950/2015) and was in compliance with all local relevant ethical regulations.

Genomic DNA was isolated from whole blood of recipients and donors (before transplantation) using the QIAcube automated system (Qiagen) at the regional centres of the Instituto Português do Sangue e Transplantação (Portugal). Genotyping of the nonsynonymous rs2306894 SNP in the CLEC1A gene was performed using KASPar assays (LGC Genomics) according to manufacturer's instructions in an Applied Biosystems 7500 Fast real-time PCR system (Thermo Fisher). Genotyping sets included randomly selected replicates of previously typed samples, and agreement between original and duplicate samples was $\geq 99 \%$. Peripheral blood mononuclear cells from healthy genotyped donors were enriched from buffy coats using Histopaque ${ }^{\circledR}-1077$ (Sigma-Aldrich) and contaminating erythrocytes removed using Red Blood Cell Lysis Buffer (Sigma-Aldrich).

Participants gave written informed consent prior to blood collection. Monocytes were isolated by positive selection using magnetically labelled CD14+ MicroBeads (Miltenyi Biotec) on a MiniMACS separator and seeded at 106 cells $/ \mathrm{mL}$ in 24-well plates for 7 days in RPMI-1640 medium supplemented with $10 \%(\mathrm{v} / \mathrm{v})$ human serum and $20 \mathrm{ng} / \mathrm{mL}$ recombinant human granulocyte macrophage colonystimulating factor (GM-CSF, Gibco). Acquisition of macrophage morphology was confirmed by phase contrast microscopy (Axiovert 135, Zeiss). For infection, macrophages were washed and then infected with live conidia of A. fumigatus strain $\mathrm{A} 1163$ at a ratio of $1: 10$ (cells/fungus) for $20 \mathrm{~h}$ at $37^{\circ} \mathrm{C}$ and $5 \% \mathrm{CO} 2$. Cytokines in supernatants were detected using DuoSet ELISA systems (BioLegend), according to the manufacturer's instructions. At least two technical replicates were performed for each donor.

For the independent cohort, genomic DNA was isolated from EDTA venous blood of healthy Dutch volunteers using the Gentra Pure Gene Blood kit (Qiagen, Venlo, The Netherlands) and genotyped for CLEC1A polymorphisms using the Illumina Immunochip SNP array platform, described previously42. Participants gave written informed consent prior to blood collection. As the CLEC1A SNP of interest (exonic rs2306894) was not represented on the genotyping platform, two intronic polymorphisms (rs7972187 and rs3825300) were used as markers. Linkage analysis revealed complete linkage disequilibrium of these two polymorphisms with the SNP of interest43 (R2=1). 105 PBMCs isolated from genotyped donors were stimulated with 107 killed conidia/mL of the clinical isolate A. fumigatus V05-2744. After $24 \mathrm{~h}$ incubation in the presence of $10 \%$ human pooled serum at $37^{\circ} \mathrm{C}$ and $5 \%$ CO2 supernatants were collected and IL-1 $\beta$ and IL- 8 were measured by ELISA (R\&D Systems, UK, and PeliKine, Sanquin, The Netherlands, respectively).

The probability of invasive aspergillosis resulting from CLEC1A rs2306894 SNP was analysed using the cumulative incidence method and compared using Gray's test45. Cumulative incidences were computed with the cmprsk package for $R$ version 2.10.146, with censoring of data at the date of last follow-up visit and defining relapse and death as competing events. A period of 24 months after transplant was chosen to include all cases of fungal infection. Murine survival data were analysed with the log rank test using GraphPad Prism. No data was excluded. 


\section{natureresearch}

\section{Flow Cytometry Reporting Summary}

Form fields will expand as needed. Please do not leave fields blank.

\section{- Data presentation}

For all flow cytometry data, confirm that:

$\bigotimes 1$. The axis labels state the marker and fluorochrome used (e.g. CD4-FITC).

2. The axis scales are clearly visible. Include numbers along axes only for bottom left plot of group (a 'group' is an analysis of identical markers).

$\bigotimes$ 3. All plots are contour plots with outliers or pseudocolor plots.

$\bigotimes 4$. A numerical value for number of cells or percentage (with statistics) is provided.

\section{- Methodological details}

5. Describe the sample preparation.

\section{Murine cells and tissues}

Murine peripheral blood leucocytes, resident and thioglycolate-elicited inflammatory peritoneal cells, alveolar macrophages and bone marrow cells were isolated essentially as described previously (Taylor PR, Eur J Immunol, 2003). For platelets, peripheral blood was collected in $3.8 \%(\mathrm{w} / \mathrm{v})$ sodium citrate buffer and centrifuged at $200 \times \mathrm{xg}$ at $25^{\circ} \mathrm{C}$. The supernatant, containing platelet rich plasma, was used for subsequent analysis. Bone marrow-derived macrophages or dendritic cells, generated using L929 conditioned medium or $20 \mathrm{ng} / \mathrm{mL}$ GM-CSF (R\&D Systems), respectively, were prepared as described (Pyz E, Eur J Immunol, 2008). In some experiments, cells were stimulated with $100 \mathrm{ng} / \mathrm{mL}$ lipopolysaccharide from Escherichia coli (Sigma).

Tissues isolated from mice were cut into small pieces and incubated for $30 \mathrm{~min}$ at $37^{\circ} \mathrm{C}$ with Liberase (Roche) and DNAse (Roche) in RPMI (Gibco), except for the small intestine which was incubated with Collagenase VIII (Sigma-Aldrich). Cells were disaggregated using the gentleMACS ${ }^{\mathrm{TM}}$ Dissociator (Miltenyi), strained through $70 \mu \mathrm{m}$ nylon cell strainers (Fisher Scientific) and collected by centrifugation. Red blood cells were removed using Pharm Lyse (BD Biosciences).

NIH3T3 fibroblasts stably expressing full length murine or human MelLec were maintained at $37^{\circ} \mathrm{C}$ and $5 \% \mathrm{CO} 2$ in DMEM or RPMI medium supplemented with $10 \%$ heat-inactivated foetal calf serum, 100 units $/ \mathrm{mL}$ penicillin, $0.1 \mathrm{mg} / \mathrm{mL}$ streptomycin, and $2 \mathrm{mM} \mathrm{L-glutamine.}$

For flow cytometry, isolated cells were washed in FACS wash (PBS with $0.5 \%(\mathrm{w} / \mathrm{v}$ ) BSA and 5-10 mM EDTA) containing anti-CD16/CD32 (Clone 2.4G2, prepared in house). The following antibodies (all from BD Biosciences, eBioscience or Abcam) were used for FACS analysis following standard methodology: anti-MelLec-Biotin, Streptavidin-PE-CF594, anti-CD45.2-FITC (Clone 104), biotinylated anti-CD61 (clone 2C9.G2), anti-CD326-APC (EpCAM; Clone G8.8), anti-CD31-PE-Cyanine7 (PECAM-1; Clone 390), Ly6G-APC (clone 1A8), CD11b-PE-Cy7 (clone M1/70), CD11c-PerCPCy5.5 (clone HL3), Siglec-F-BV421 (clone E50-2440) and F4/80-AF700 (clone $\mathrm{CL}: \mathrm{A} 3-1)$ and relevant isotype controls. Cell viability was detected using the fixable viability dye efluor-780 (eBioscience) in PBS, and the cells were fixed with $1 \%$ formaldehyde prior to acquisition.

Fungal strains

For flow cytometry, fungi were incubated in 3\% (w/v) BSA in PBS and Fc-proteins were added to a final concentration of $5 \mu \mathrm{g} / \mathrm{mL}$. Following incubation at $4^{\circ} \mathrm{C}$, fungal 
6. Identify the instrument used for data collection.

7. Describe the software used to collect and analyze the flow cytometry data.

8. Describe the abundance of the relevant cell populations within post-sort fractions.

9. Describe the gating strategy used. particles were washed in FACS buffer (0.5\% (w/v) BSA and 2 mM EDTA in PBS), and bound Fc-proteins detected with phycoerythrin-conjugated donkey anti-human antibody (Jackson ImmunoResearch), fixed in 1\% (v/v) formaldehyde, and analysed.

Becton Dickinson LSRII, LSR Fortessa or FACS Calibur cell analyzer.

For collection Becton Dickinson FACSDiva software was used. For analysis FlowJo software was used.

No cell populations were sorted for this manuscript.

Gating strategy for fungal staining

Using the FSC/SSC plot, a gate was drawn to select fungal cells and to exclude debris. Using SSC/PE plot histogram overlays were made of Fc-MelLec vs Fc-control for various fungi.

Gating strategy for murine tissue staining

Using the FSC/SSC plot, a gate was drawn to select cells and exclude debris. Using the SSC/Efluor780 plot, a gate was drawn to select viable cells. Using the SSC/FITC plot, we gated on CD45 negative and positive cells. In the CD45- population, we gated on CD31 negative or positive cells as well as EpCAM negative and positive cells. All subpopulations were assessed for presence of MelLec by histogram overlay of the SSC/PECF594 plot for anti-MelLec or isotype.

Gating strategy for BAL

Using the FSC/SSC plot, a gate was drawn to select cells and exclude debris. Using the SSC/CD45FITC plot, we gated on CD45 positive cells. In the CD45 positive population, neutrophils (CD11b+Ly6G+), alveolar macrophages (CD11c+SiglecF+), inflammatory macrophages $(C D 11 b+F 480+)$ and eosinophils (CD11b+SiglecF+) were determined.

Gating strategy for NIH cells

Using the FSC/SSC plot, a gate was drawn to select NIH cells and to exclude debris. Using SSC/PE-CF594 plot histogram overlays were made of anti-MelLec staining on $\mathrm{NIH}-\mathrm{MelLec}$ vs NIH parental cells.

Positive and negative populations for gating were defined using FMO and isotype controls.

Tick this box to confirm that a figure exemplifying the gating strategy is provided in the Supplementary Information. $\bigotimes$ 